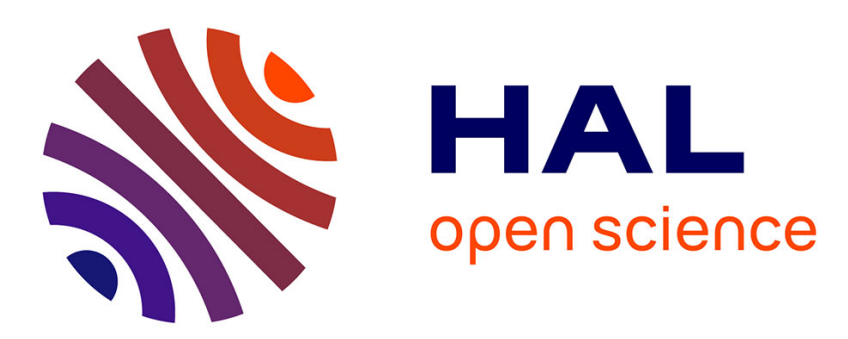

\title{
On some ordinal models for decision making under uncertainty
}

Denis Bouyssou, Marc Pirlot

\section{To cite this version:}

Denis Bouyssou, Marc Pirlot. On some ordinal models for decision making under uncertainty. pp.37, 2004. hal-00018253

\section{HAL Id: hal-00018253 https://hal.science/hal-00018253}

Submitted on 31 Jan 2006

HAL is a multi-disciplinary open access archive for the deposit and dissemination of scientific research documents, whether they are published or not. The documents may come from teaching and research institutions in France or abroad, or from public or private research centers.
L'archive ouverte pluridisciplinaire $\mathbf{H A L}$, est destinée au dépôt et à la diffusion de documents scientifiques de niveau recherche, publiés ou non, émanant des établissements d'enseignement et de recherche français ou étrangers, des laboratoires publics ou privés. 


\title{
On some ordinal models for decision making under uncertainty
}

\author{
Denis Bouyssou*, Marc Pirlot ${ }^{\dagger}$
}

\begin{abstract}
In the field of Artificial Intelligence many models for decision making under uncertainty have been proposed that deviate from the traditional models used in Decision Theory, i.e. the Subjective Expected Utility (SEU) model and its many variants. These models aim at obtaining simple decision rules that can be implemented by efficient algorithms while based on inputs that are less rich than what is required in traditional models. One of these models, called the likely dominance (LD) model, consists in declaring that an act is preferred to another as soon as the set of states on which the first act gives a better outcome than the second act is judged more likely than the set of states on which the second act is preferable. The LD model is at much variance with the SEU model. Indeed, it has a definite ordinal flavor and it may lead to preference relations between acts that are not transitive. This paper proposes a general model for decision making under uncertainty tolerating intransitive and/or incomplete preferences that will contain both the SEU and the LD models as particular cases. Within the framework of this general model, we propose a characterization of the preference relations that can be obtained with the LD model. This characterization shows that the main distinctive feature of such relations lies in the very poor relation comparing preference differences that they induce on the set of outcomes.
\end{abstract}

Key words : Decision under uncertainty, Subjective Expected Utility, Conjoint measurement, Nontransitive preferences, Likely Dominance model.

*LAMSADE, Université Paris-Dauphine, Place du Maréchal de Lattre de Tassigny, 75775 Paris cedex 16, France. bouys sou@lamsade. dauphine. fr

†Faculté Polytechnique de Mons, 9, rue de Houdain, B-7000 Mons, Belgium. marc.pirlot@fpms. ac.be 


\section{Introduction}

The specific needs of Artificial Intelligence techniques have led many Computer Scientists to propose models for decision under uncertainty that are at variance with the classical models used in Decision Theory, i.e. the Subjective Expected Utility (SEU) model and its many variants (see Fishburn, 1988; Wakker, 1989, for overviews). This gives rise to what is often called "qualitative decision theory" (see Boutilier, 1994; Brafman and Tennenholtz, 1997, 2000; Doyle and Thomason, 1999; Dubois et al., 1997, 2001; Lehmann, 1996; Tan and Pearl, 1994, for overviews). These models aim at obtaining simple decision rules that can be implemented by efficient algorithms while based on inputs that are less rich than what is required in traditional models. This can be achieved, e.g. comparing acts only on the basis of their consequences in the most plausible states (Boutilier, 1994; Tan and Pearl, 1994) or refining the classical criteria (Luce and Raiffa, 1957; Milnor, 1954) for decision making under complete ignorance (see Brafman and Tennenholtz, 2000; Dubois et al., 2001).

One such model, called the "likely dominance" (LD) model, was recently proposed by Dubois et al. (1997) and later studied in Dubois et al. (2003a, 2002) and Fargier and Perny (1999). It consists in declaring that an act $a$ is preferred to an act $b$ as soon as the set of states for which $a$ gives a better outcome than $b$ is judged "more likely" than the set of states for which $b$ gives a better outcome than $a$. Such a way of comparing acts has a definite ordinal flavor. It rests on a simple "voting" analogy and can be implemented as soon as a preference relation on the set of outcomes and a likelihood relation between subsets of states (i.e. events) are known. Contrary to the other models mentioned above, simple examples inspired from Condorcet's paradox (see Sen, 1986) show that the LD model does not always lead to preference relations between acts that are complete or transitive. Such relations are therefore quite different from the ones usually dealt with in Decision Theory.

Previous characterizations (see Dubois et al., 2003a, 2002; Fargier and Perny, 1999) of the relations that can be obtained using the LD model (of, for short, LD relations) have emphasized their "ordinal" character via the use of variants of a "noncompensation" condition introduced in Fishburn $(1975,1976,1978)$ that have been thoroughly studied in the area of multiple criteria decision making (see Bouyssou, 1986, 1992; Bouyssou and Vansnick, 1986; Dubois et al., 2003b; Fargier and Perny, 2001; Vansnick, 1986). Since this condition is wholly specific to such relations, these characterizations are not perfectly suited to capture their essential distinctive features within a more general framework that would also include more traditional preference relations.

The purpose of this paper is twofold. We first introduce a general axiomatic framework for decision under uncertainty that will contain both the SEU and LD models as particular cases. This general framework tolerating incomplete and/or intransitive preferences is based on related work in the area of conjoint measurement (see Bouyssou and 
Pirlot, 2002). The second aim of this paper is to propose an alternative characterization of the preference relations that can be obtained using the likely dominance rule within this general framework. This characterization will allow us to emphasize the main distinctive feature of such relations, i.e. the poor relation comparing preference differences that they induce on the set of outcomes. This analysis specializes the one in Bouyssou and Pirlot (2004b) to the case of decision making under uncertainty.

It should be noticed that the interest of studying models tolerating intransitive preferences was forcefully argued by Fishburn (1991). It has already generated much work (see, e.g. Fishburn, 1982, 1984, 1988, 1989, 1990, 1991; Fishburn and Lavalle, 1987a,b, 1988; Lavalle and Fishburn, 1987; Loomes and Sugden, 1982; Nakamura, 1998; Sugden, 1993). These models all use some form of an additive nontransitive model. The originality of our approach is to replace additivity by a mere decomposability requirement which, at the cost of much weaker uniqueness results, allows for a very simple axiomatic treatment.

This paper is organized as follows. Section 2 introduces our setting and notation. The LD model is introduced in section 3. Our general framework for decision making under uncertainty is presented and analyzed in section 4. Section 5 characterize the relations that can be obtained using the LD model within our general framework. A final section discusses our results and presents several extensions of our analysis. An appendix contains examples showing the independence of the conditions used in the paper. The rest of this section is devoted to our, classical, vocabulary concerning binary relations.

A binary relation $\mathcal{R}$ on a set $X$ is a subset of $X \times X$; we write $a \mathcal{R} b$ instead of $(a, b) \in \mathcal{R}$. A binary relation $\mathcal{R}$ on $X$ is said to be:

- reflexive if $[a \mathcal{R} a]$,

- complete if $[a \mathcal{R} b$ or $b \mathcal{R} a]$,

- symmetric if $[a \mathcal{R} b] \Rightarrow[b \mathcal{R} a]$,

- asymmetric if $[a \mathcal{R} b] \Rightarrow[\operatorname{Not}[b \mathcal{R} a]]$,

- transitive if $[a \mathcal{R} b$ and $b \mathcal{R} c] \Rightarrow[a \mathcal{R} c]$,

- Ferrers if $[(a \mathcal{R} b$ and $c \mathcal{R} d) \Rightarrow(a \mathcal{R} d$ or $c \mathcal{R} b)]$,

- semi-transitive if $[(a \mathcal{R} b$ and $b \mathcal{R} c) \Rightarrow(a \mathcal{R} d$ or $d \mathcal{R} c)]$

for all $a, b, c, d \in X$.

A weak order (resp. an equivalence) is a complete and transitive (resp. reflexive, symmetric and transitive) binary relation. If $\mathcal{R}$ is an equivalence on $X, X / \mathcal{R}$ will denote the set of equivalence classes of $\mathcal{R}$ on $X$. An interval order is a complete and Ferrers binary relation. A semiorder is a semi-transitive interval order. 


\section{The setting}

We adopt a classical setting for decision under uncertainty with a finite number of states. Let $\Gamma=\{\alpha, \beta, \gamma, \ldots\}$ be the set of outcomes and $N=\{1,2, \ldots, n\}$ be the set of states. It is understood that the elements of $N$ are exhaustive and mutually exclusive: one and only one state will turn out to be true. An act is a function from $N$ to $\Gamma$. The set of all acts is denoted by $\mathcal{A}=\Gamma^{N}$. Acts will be denoted by lowercase letters $a, b, c, d, \ldots$. An act $a \in \mathcal{A}$ therefore associates to each state $i \in N$ an outcome $a(i) \in \Gamma$. We often abuse notation and write $a_{i}$ instead of $a(i)$.

Among the elements of $\mathcal{A}$ are constant acts, i.e. acts giving the same outcome in all states. We denote $\bar{\alpha}$ the constant act giving the outcome $\alpha \in \Gamma$ in all states $i \in N$. Let $E \subseteq N$ and $a, b \in \mathcal{A}$. We denote $a_{E} b$ the act $c \in \mathcal{A}$ such that $c_{i}=a_{i}$, for all $i \in E$ and $c_{i}=b_{i}$, for all $i \in N \backslash E$. Similarly $\alpha_{E} b$ will denote the act $d \in \mathcal{A}$ such that $d_{i}=\alpha$, for all $i \in E$ and $d_{i}=b_{i}$, for all $i \in N \backslash E$. When $E=\{i\}$ we write $a_{i} b$ and $\alpha_{i} b$ instead of $a_{\{i\}} b$ and $\alpha_{\{i\}} b$.

In this paper $\succsim$ will always denote a binary relation on the set $\mathcal{A}$. The binary relation $\succsim$ is interpreted as an "at least as good as" preference relation between acts. We note $\succ$ (resp. $\sim$ ) the asymmetric (resp. symmetric) part of $\succsim$. A similar convention holds when $\succsim$ is starred, superscripted and/or subscripted. The relation $\succsim$ induces a relation $\succsim_{\Gamma}$ on the set $\Gamma$ of outcomes via the comparison of constant acts letting:

$$
\alpha \succsim_{\Gamma} \beta \Leftrightarrow \bar{\alpha} \succsim \bar{\beta}
$$

Let $E$ be a nonempty subset of $N$. We define the relation $\succsim_{E}$ on $\mathcal{A}$ letting, for all $a, b \in \mathcal{A}$,

$$
a \succsim_{E} b \Leftrightarrow\left[a_{E} c \succsim b_{E} c, \text { for all } c \in \mathcal{A}\right]
$$

When $E=\{i\}$ we write $\succsim_{i}$ instead of $\succsim_{\{i\}}$.

If, for all $a, b \in \mathcal{A}, a_{E} c \succsim b_{E} c$, for some $c \in \mathcal{A}$, implies $a \succsim_{E} b$, we say that $\succsim$ is independent for $E$. If $\succsim$ is independent for all nonempty subsets of states we say that $\succsim$ is independent. It is not difficult to see that a binary relation is independent if and only if it is independent for $N \backslash\{i\}$, for all $i \in N$ (see Wakker, 1989). Independence as defined here is therefore nothing else than the Sure Thing Principle (postulate P2) introduced by Savage (1954).

We say that state $i \in N$ is influent (for $\succsim$ ) if there are $\alpha, \beta, \gamma, \delta \in \Gamma$ and $a, b \in \mathcal{A}$ such that $\alpha_{i} a \succsim \beta_{i} b$ and $\operatorname{Not}\left[\gamma_{i} a \succsim \delta_{i} b\right]$ and degenerate otherwise. It is clear that a degenerate state has no influence whatsoever on the comparison of the elements of $\mathcal{A}$ and may be suppressed from $N$. In order to avoid unnecessary minor complications, we suppose henceforth that all states in $N$ are influent. Note that this does not rule out the existence 
of null events $E \subseteq N$, i.e. such that $a_{E} c \sim b_{E} c$, for all $a, b, c \in \mathcal{A}$. This is exemplified below.

\section{Example 1}

Let $N=\{1,2,3,4\}$ and $\Gamma=\mathbb{R}$. Let $p_{1}=p_{2}=p_{3}=p_{4}=1 / 4$. Define $\succsim$ on $\mathcal{A}$ letting

$$
a \succsim b \Leftrightarrow \sum_{i \in S(a, b)} p_{i} \geq \sum_{j \in S(b, a)} p_{j}-1 / 4
$$

for all $a, b \in \mathcal{A}$, where $S(a, b)=\left\{i \in N: a_{i} \geq b_{i}\right\}$. With such a relation, it is easy to see that all states are influent while they are all null. Observe that $\succsim$ is complete but is not transitive. We shall shortly see that this relation can be obtained with the LD model.

\section{The likely dominance model}

The following definition, building on Dubois et al. (1997) and Fargier and Perny (1999), formalizes the idea of a LD relation, i.e., of a preference relation that has been obtained comparing acts by pairs on the basis of the "likelihood" of the states favoring each element of the pair.

\section{Definition 1 (LD relations)}

Let $\succsim$ be a reflexive binary relation on $\mathcal{A}$. We say that $\succsim i s ~ a ~ L D$ relation if there are:

- a complete binary relation $\mathcal{S}$ on $\Gamma$,

- a binary relation $\unrhd$ between subsets of $N$ having $N$ for union that is monotonic w.r.t. inclusion, i.e. such that for all $A, B, C, D \subseteq N$,

$$
[A \unrhd B, C \supseteq A, B \supseteq D, C \cup D=N] \Rightarrow C \unrhd D
$$

such that, for all $a, b \in \mathcal{A}$,

$$
a \succsim b \Leftrightarrow \mathcal{S}(a, b) \unrhd \mathcal{S}(b, a),
$$

where $\mathcal{S}(a, b)=\left\{i \in N: a_{i} \mathcal{S} b_{i}\right\}$. We say that $\langle\unrhd, \mathcal{S}\rangle$ is a representation of $\succsim$.

Hence, when $\succsim$ is a LD relation, the preference between $a$ and $b$ only depends on the subsets of states favoring $a$ or $b$ in terms of the complete relation $\mathcal{S}$. It does not depend on "preference differences" between outcomes besides what is indicated by $\mathcal{S}$. A major advantage of the LD model is that it can be applied to compare acts as soon as there is a binary relation allowing to compare outcomes and a relation allowing to compare events in terms of likelihood. 
Let $\succsim$ be a LD relation with a representation $\langle\underline{Q}, \mathcal{S}\rangle$. We denote by $\mathcal{J}$ (resp. $\mathcal{P}$ ) the symmetric part (resp. asymmetric part) of $\mathcal{S}$. For all $A, B \subseteq N$, we define the relations $\triangleq$, $\triangleright$ and $\bowtie$ between subsets of $N$ having $N$ for union letting: $A \triangleq B \Leftrightarrow[A \unrhd B$ and $B \unrhd$ $A], A \triangleright B \Leftrightarrow[A \unrhd B$ and $\operatorname{Not}[B \unrhd A]], A \bowtie B \Leftrightarrow[\operatorname{Not}[A \unrhd B]$ and $\operatorname{Not}[B \unrhd A]]$.

The following lemma takes note of some elementary properties of LD relations; it uses the hypothesis that all states are influent.

\section{Lemma 1}

If $\succsim$ is a $L D$ relation with a representation $\langle\unrhd, \mathcal{S}\rangle$, then:

1. $\mathcal{P}$ is nonempty,

2. for all $A, B \subseteq N$ such that $A \cup B=N$ exactly one of $A \triangleright B, B \triangleright A, A \triangleq B$ and $A \bowtie B$ holds and we have $N \triangleq N$,

3. for all $A \subseteq N, N \unrhd A$,

4. $N \triangleright \varnothing$,

5. $\succsim$ is independent,

6. ¿ is marginally complete, i.e., for all $i \in N$, all $\alpha, \beta \in \Gamma$ and all $a \in \mathcal{A}, \alpha_{i} a \succsim \beta_{i} a$ or $\beta_{i} a \succsim \alpha_{i} a$,

7. $\mathcal{S}=\succsim_{\Gamma}$,

8. for all $i \in N$ and all $a, b \in \mathcal{A}$, either $a \succsim_{i} b \Leftrightarrow a_{i} \mathcal{S} b_{i}$ or $a \sim_{i} b$,

9. $\succsim$ has a unique representation.

\section{PROOF}

Part 1. If $\mathcal{P}$ is empty, then, since $\mathcal{S}$ is complete, $\mathcal{S}(a, b)=N$, for all $a, b \in \mathcal{A}$. Hence, for all $i \in N$, all $\alpha, \beta, \gamma, \delta \in \Gamma$, and all $a, b \in \mathcal{A}$,

$$
\begin{aligned}
& \mathcal{S}\left(\alpha_{i} a, \beta_{i} b\right)=\mathcal{S}\left(\gamma_{i} a, \delta_{i} b\right) \text { and } \\
& \mathcal{S}\left(\beta_{i} b, \alpha_{i} a\right)=\mathcal{S}\left(\delta_{i} b, \gamma_{i} a\right) .
\end{aligned}
$$

This implies, using (2), that state $i \in N$ is degenerate, contrarily to our hypothesis.

Part 2. Since the relation $\mathcal{P}$ is nonempty and $\mathcal{S}$ is complete, for all $A, B \subseteq N$ such that $A \cup B=N$, there are $a, b \in \mathcal{A}$ such that $\mathcal{S}(a, b)=A$ and $\mathcal{S}(b, a)=B$. We have, by construction, exactly one of $a \succ b, b \succ a, a \sim b$ and $[\operatorname{Not}[a \succsim b]$ and $\operatorname{Not}[b \succsim a]]$. Hence, using (2), we have exactly one of $A \triangleright B, B \triangleright A, A \triangleq B$ and $A \bowtie B$. Since the relation $\mathcal{S}$ is complete, we have $\mathcal{S}(a, a)=N$. Using the reflexivity of $\succsim$, we know that $a \sim a$, so that (2) implies $N \triangleq N$. 
Parts 3 and 4. Let $A \subseteq N$. Because $N \triangleq N$, the monotonicity of $\unrhd$ implies $N \unrhd A$. Suppose that $\varnothing \unrhd N$. Then the monotonicity of $\unrhd$ would imply that $A \unrhd B$, for all $A, B \subseteq N$ such that $A \cup B=N$. This would contradict the fact that each state is influent.

Part 5. Using the completeness of $\mathcal{S}$, we have, for all $\alpha, \beta, \gamma, \delta \in \Gamma$ and all $a, b \in \mathcal{A}$,

$$
\begin{aligned}
& \mathcal{S}\left(\alpha_{i} a, \alpha_{i} b\right)=\mathcal{S}\left(\beta_{i} a, \beta_{i} b\right) \text { and } \\
& \mathcal{S}\left(\alpha_{i} b, \alpha_{i} a\right)=\mathcal{S}\left(\beta_{i} b, \beta_{i} a\right) .
\end{aligned}
$$

Using (2), this implies that, for all $i \in N$, all $\alpha, \beta \in \Gamma$ and all $a, b \in \mathcal{A}, \alpha_{i} a \succsim \alpha_{i} b \Leftrightarrow$ $\beta_{i} a \succsim \beta_{i} b$. Therefore, $\succsim$ is independent for $N \backslash\{i\}$ and, hence, independent.

Part 6 follows from the fact that $\mathcal{S}$ is complete, $N \triangleq N$ and $N \unrhd N \backslash\{i\}$, for all $i \in N$.

Part 7. Suppose that $\alpha \succsim_{\Gamma} \beta$ so that $\bar{\alpha} \bar{\beta}_{\beta}$ and $\operatorname{Not}[\alpha \mathcal{S} \beta]$. Since $\mathcal{S}$ is complete, we have $\beta \mathcal{P} \alpha$. Using (2) and $N \triangleright \varnothing$, we have $\bar{\beta} \succ \bar{\alpha}$, a contradiction. Conversely, if $\alpha \mathcal{S} \beta$ we obtain, using (2) and the fact that $N \unrhd A$, for all $A \subseteq N, \bar{\alpha} \succsim_{\beta}$ so that $\alpha \succsim_{\Gamma} \beta$.

Part 8. Let $i \in N$. We know that $N \triangleq N$ and $N \unrhd N \backslash\{i\}$. If $N \triangleq N \backslash\{i\}$, then (2) implies $a \succsim_{i} b$ for all $a, b \in \mathcal{A}$. Otherwise we have $N \triangleright N \backslash\{i\}$ and $N \triangleq N$. It follows that $\alpha \mathcal{S} \beta \Rightarrow \bar{\alpha} \succsim_{i} \bar{\beta}$ and $\alpha \mathcal{P} \beta \Rightarrow \bar{\alpha} \succ_{i} \bar{\beta}$. Since $\mathcal{S}$ and $\succsim_{i}$ are complete, it follows that $\mathcal{S}=\succsim_{i}$.

Part 9. Suppose that $\succsim$ is a LD relation with a representation $\langle\unrhd, \mathcal{S}\rangle$. Suppose that $\succsim$ has another representation $\left\langle\underline{ }^{\prime}, \mathcal{S}^{\prime}\right\rangle$. Using part 7, we know that $\mathcal{S}=\mathcal{S}^{\prime}=\succsim_{\Gamma}$. Using (2), it follows that $\unrhd=\unrhd^{\prime}$.

\section{A general framework for decision under uncertainty tolerating intransitive preferences}

We consider in this section binary relations $\succsim$ on $\mathcal{A}$ that can be represented as:

$$
a \succsim b \Leftrightarrow F\left(p\left(a_{1}, b_{1}\right), p\left(a_{2}, b_{2}\right), \ldots, p\left(a_{n}, b_{n}\right)\right) \geq 0
$$

where $p$ is a real-valued function on $\Gamma^{2}$ that is skew symmetric (i.e. such that $p(\alpha, \beta)=$ $-p(\beta, \alpha)$, for all $\alpha, \beta \in \Gamma)$ and $F$ is a real-valued function on $\prod_{i=1}^{n} p\left(\Gamma^{2}\right)$ being nondecreasing in all its arguments and such that, abusing notation, $F(\mathbf{0}) \geq 0$.

It is useful to interpret $p$ as a function measuring preference differences between outcomes. The fact that $p$ is supposed to be skew symmetric means that the preference difference between $\alpha$ and $\beta$ is the opposite of the preference difference between $\beta$ and $\alpha$, which seems a reasonable hypothesis for preference differences. With this interpretation 
in mind, the acts $a$ and $b$ are compared as follows. In each state $i \in N$, the preference difference between $a_{i}$ and $b_{i}$ is computed. The synthesis of these preference differences is performed applying the function $F$. If this synthesis is positive, we conclude that $a \succsim b$. Given this interpretation, it seems reasonable to suppose that $F$ is nondecreasing in each of its arguments. The fact that $F(\mathbf{0}) \geq 0$ simply means that the synthesis of null preference differences in each state should be nonnegative; this ensures that $\succsim$ will be reflexive. Model (UM) is the specialization to the case of decision making under uncertainty of conjoint measurement models studied in Bouyssou and Pirlot (2002).

It is not difficult to see that model (UM) encompasses preference relations $\succsim$ on $\mathcal{A}$ that are neither transitive nor complete. It is worth noting that this model is sufficiently flexible to contain many others as particular cases including:

- the SEU model (see, e.g. Wakker, 1989) in which:

$$
a \succsim b \Leftrightarrow \sum_{i=1}^{n} w_{i} u\left(a_{i}\right) \geq \sum_{i=1}^{n} w_{i} u\left(b_{i}\right)
$$

where $w_{i}$ are nonnegative real numbers that add up to one and $u$ is a real-valued function on $\Gamma$,

- the Skew Symmetric Additive model (SSA) (see Fishburn, 1988, 1990) in which

$$
a \succsim b \Leftrightarrow \sum_{i=1}^{n} w_{i} \Phi\left(a_{i}, b_{i}\right) \geq 0
$$

where $w_{i}$ are nonnegative real numbers that add up to one and $\Phi$ is a skew symmetric $(\Phi(\alpha, \beta)=-\Phi(\beta, \alpha))$ real-valued function on $\Gamma^{2}$.

We will show in the next section that model (UM) also contains all LD relations. As shown below, model (UM) implies that $\succsim$ is independent. It is therefore not suited to cope with violations of the Sure Thing Principle that have been widely documented in the literature (Allais, 1953; Ellsberg, 1961; Kahneman and Tversky, 1979), which can be done, e.g. using Choquet Expected Utility or Cumulative Prospect Theory (see Chew and Karni, 1994; Gilboa, 1987; Karni and Schmeidler, 1991; Luce, 2000; Nakamura, 1990; Schmeidler, 1989; Wakker, 1989, 1994, 1996; Wakker and Tversky, 1993).

The flexibility of model (UM) may obscure some of its properties. We summarize what appears to be the most important ones in the following.

\section{Lemma 2}

Let $\succsim$ be a binary relation on $\mathcal{A}$ that has a representation in model (UM). Then:

1. $\succsim$ is reflexive, independent and marginally complete, 
2. $\left[a \succ_{i} b\right.$ for all $\left.i \in J \subseteq N\right] \Rightarrow\left[a \succ_{J} b\right]$,

3. $\succsim_{\Gamma}$ is complete.

\section{PROOF}

Part 1 . The reflexivity of $\succsim$ follows from the skew symmetry of $p$ and $F(\mathbf{0}) \geq 0$. Independence follows from the fact that $p(\alpha, \alpha)=0$, for all $\alpha \in \Gamma$. Not $\left[\alpha_{i} a \succsim \beta_{i} a\right]$ and $N o t\left[\beta_{i} a \succsim \alpha_{i} a\right]$ imply, abusing notation, $F\left([p(\alpha, \beta)]_{i},[\mathbf{0}]_{-i}\right)<0$ and $F\left([p(\beta, \alpha)]_{i},[\mathbf{0}]_{-i}\right)$ $<0$. Since $F(0) \geq 0$ and $F$ is nondecreasing, we have $p(\alpha, \beta)<0$ and $p(\beta, \alpha)<0$, which contradicts the skew symmetry of $p$. Hence, $\succsim$ is marginally complete.

Part 2. Observe that $\alpha \succ_{i} \beta$ is equivalent to $F\left([p(\alpha, \beta)]_{i},[0]_{-i}\right) \geq 0$ and $F\left([p(\beta, \alpha)]_{i}\right.$, $\left.[0]_{-i}\right)<0$. Since $F(\mathbf{0}) \geq 0$ we know that $p(\beta, \alpha)<0$ using the nondecreasingness of $F$. The skew symmetry of $p$ implies $p(\alpha, \beta)>0>p(\beta, \alpha)$ and the desired property easily follows using the nondecreasingness of $F$.

Part 3. Because $p$ is skew symmetric, we have, for all $\alpha, \beta \in \Gamma, p(\alpha, \beta) \geq 0$ or $p(\beta, \alpha) \geq 0$. Since $F(\mathbf{0}) \geq 0$, the completeness of $\succsim_{\Gamma}$ follows from the nondecreasingness of $F$.

The analysis of model (UM) heavily rests on the study of induced relations comparing preference differences on the set of outcomes. The interest of such relations was already powerfully stressed by Wakker $(1988,1989)$ (note however that, although we use similar notation, our definitions differs from his).

\section{Definition 2 (Relations comparing preference differences)}

Let $\succsim$ be a binary relation on $\mathcal{A}$. We define the binary relations $\succsim^{*}$ and $\succsim^{* *}$ on $\Gamma^{2}$ letting, for all $\alpha, \beta, \gamma, \delta \in \Gamma$,

$$
\begin{gathered}
(\alpha, \beta) \succsim^{*}(\gamma, \delta) \Leftrightarrow\left[\text { for all } a, b \in \mathcal{A} \text { and all } i \in N, \gamma_{i} a \succsim \delta_{i} b \Rightarrow \alpha_{i} a \succsim \beta_{i} b\right] \\
(\alpha, \beta) \succsim^{* *}(\gamma, \delta) \Leftrightarrow\left[(\alpha, \beta) \succsim^{*}(\gamma, \delta) \text { and }(\delta, \gamma) \succsim^{*}(\beta, \alpha)\right] .
\end{gathered}
$$

The asymmetric and symmetric parts of $\succsim^{*}$ are respectively denoted by $\succ^{*}$ and $\sim^{*}$, a similar convention holding for $\succsim^{* *}$. By construction, $\succsim^{*}$ and $\succsim^{* *}$ are reflexive and transitive. Therefore, $\sim^{*}$ and $\sim^{* *}$ are equivalence relations. Note that, by construction, $\succsim^{* *}$ is reversible, i.e. $(\alpha, \beta) \succsim^{* *}(\gamma, \delta) \Leftrightarrow(\delta, \gamma) \succsim^{* *}(\beta, \alpha)$.

We note a few useful connections between $\succsim^{*}$ and $\succsim$ in the following lemma.

\section{Lemma 3}

1. $\succsim$ is independent if and only if (iff) $(\alpha, \alpha) \sim^{*}(\beta, \beta)$, for all $\alpha, \beta \in \Gamma$ 
2. For all $a, b, c, d \in \mathcal{A}$, all $i \in N$ and all $\alpha, \beta \in \Gamma$

$$
\begin{gathered}
{\left[a \succsim b \text { and }\left(c_{i}, d_{i}\right) \succsim^{*}\left(a_{i}, b_{i}\right)\right] \Rightarrow c_{i} a \succsim d_{i} b,} \\
{\left[\left(c_{j}, d_{j}\right) \sim^{*}\left(a_{j}, b_{j}\right), \text { for all } j \in N\right] \Rightarrow[a \succsim b \Leftrightarrow c \succsim d] .}
\end{gathered}
$$

PROOF

Part 1. It is clear that $[\succsim$ is independent $] \Leftrightarrow[\succsim$ is independent for $N \backslash\{i\}$, for all $i \in N]$. Observe that $[\succsim$ is independent for $N \backslash\{i\}$, for all $i \in N] \Leftrightarrow\left[\alpha_{i} a \succsim \alpha_{i} b \Leftrightarrow \beta_{i} a \succsim \beta_{i} b\right.$, for all $\alpha, \beta \in \Gamma$, all $i \in N$ and all $a, b \in \mathcal{A}] \Leftrightarrow\left[(\alpha, \alpha) \sim^{*}(\beta, \beta)\right.$ for all $\left.\alpha, \beta \in \Gamma\right]$.

Part 2. (3) is clear from the definition of $\succsim^{*}$, (4) follows.

The following conditions are an adaptation to the case of decision making under uncertainty of conditions used in Bouyssou and Pirlot (2002) in the context of conjoint measurement. They will prove will prove central in what follows.

\section{Definition 3 (Conditions URC1 and URC2)}

Let $\succsim$ be a binary relation on $\mathcal{A}$. This relation is said to satisfy:

$$
\begin{aligned}
\text { URC1 if } \left.\begin{array}{c}
\alpha_{i} a \succsim \beta_{i} b \\
\text { and } \\
\gamma_{j} c \succsim \delta_{j} d
\end{array}\right\} \Rightarrow\left\{\begin{array}{c}
\gamma_{i} a \succsim \delta_{i} b \\
\text { or } \\
\alpha_{i} a \succsim \beta_{i} b \\
\text { and } \beta_{j} d, \\
\beta_{j} c \succsim \alpha_{j} d
\end{array}\right\} \Rightarrow\left\{\begin{array}{c}
\gamma_{i} a \succsim \delta_{i} b \\
\text { or } \\
\delta_{j} c \succsim \gamma_{j} d,
\end{array}\right.
\end{aligned}
$$

for all $i, j \in N$, all $a, b, c, d \in \mathcal{A}$ and all $\alpha, \beta, \gamma, \delta \in \Gamma$.

Condition URC1 suggests that, independently of the state $i \in N$, either the difference $(\alpha, \beta)$ is at least as large as the difference $(\gamma, \delta)$ of vice versa. Indeed, suppose that $\alpha_{i} a \succsim$ $\beta_{i} b$ and $\operatorname{Not}\left[\gamma_{i} a \succsim \delta_{i} b\right]$. This is the sign that the preference difference between $\alpha$ and $\beta$ appears to be larger than the preference difference between $\gamma$ and $\delta$. Therefore if $\gamma_{j} c \succsim$ $\delta_{j} d$, we should have $\alpha_{j} c \succsim \beta_{j} d$, which is URC1. Similarly, condition URC2 suggests that the preference difference $(\alpha, \beta)$ is linked to the "opposite" preference difference $(\beta, \alpha)$. Indeed if $\alpha_{i} a \succsim \beta_{i} b$ and $\operatorname{Not}\left[\gamma_{i} a \succsim \delta_{i} b\right]$, so that the difference between $\gamma$ and $\delta$ is not larger than the difference between $\alpha$ and $\beta$, URC2 implies that $\beta_{j} c \succsim \alpha_{j} d$ should imply $\delta_{j} c \succsim \gamma_{j} d$, so that the difference between $\delta$ and $\gamma$ is not smaller than the difference between $\beta$ and $\alpha$. The following lemma summarizes the main consequences of URC1 and URC2.

\section{Lemma 4}

$$
\begin{aligned}
& \text { 1. } \text { URC } 1 \Leftrightarrow\left[\succsim^{*} \text { is complete }\right], \\
& \text { 2. } U R C 2 \Leftrightarrow \\
& {\left[\text { for all } \alpha, \beta, \gamma, \delta \in \Gamma, \operatorname{Not}\left[(\alpha, \beta) \succsim^{*}(\gamma, \delta)\right] \Rightarrow(\beta, \alpha) \succsim^{*}(\delta, \gamma)\right],}
\end{aligned}
$$


3. $\left[\right.$ URC1 and URC2] $\Leftrightarrow\left[\succsim^{* *}\right.$ is complete $]$.

4. In the class of reflexive relations, URC1 and URC2 are independent conditions.

5. $U R C 2 \Rightarrow[\succsim$ is independent $]$.

PROOF

Part 1. Suppose that URC1 is violated so that $\alpha_{i} a \succsim \beta_{i} b, \gamma_{j} c \succsim \delta_{j} d$, Not $\left[\gamma_{i} a \succsim \delta_{i} b\right]$ and $\operatorname{Not}\left[\alpha_{j} c \succsim \beta_{j} d\right]$. This is equivalent to $\operatorname{Not}\left[(\alpha, \beta) \succsim^{*}(\gamma, \delta)\right]$ and $\operatorname{Not}\left[(\gamma, \delta) \succsim^{*}(\alpha, \beta)\right]$.

Part 2. Suppose that URC2 is violated so that $\alpha_{i} a \succsim \beta_{i} b, \beta_{j} c \succsim \alpha_{j} d$, Not $\left[\gamma_{i} a \succsim \delta_{i} b\right]$ and $\operatorname{Not}\left[\delta_{j} c \succsim \gamma_{j} d\right]$. This is equivalent to $\operatorname{Not}\left[(\gamma, \delta) \succsim^{*}(\alpha, \beta)\right]$ and $\operatorname{Not}[(\delta, \gamma) \succsim *(\tilde{\beta}, \alpha)]$. Part 3 easily follows from parts 1 and 2 .

Part 4: see examples 2 and 3 in appendix.

Part 5. Suppose that $\alpha_{i} a \succsim \alpha_{i} b$. Using URC2 implies $\beta_{i} a \succsim \beta_{i} b$, for all $\beta \in \Gamma$. Hence, $\succsim$ is independent.

The following lemma shows that all relations satisfying model (UM) satisfy URC1 and URC2; this should be no surprise since within model (UM) the skew symmetric function $p$ induces on $\Gamma^{2}$ a reversible weak order.

\section{Lemma 5}

Let $\succsim$ be a binary relation on $\mathcal{A}$. If $\succsim$ has a representation in model (UM) then $\succsim$ satisfies $U R C 1$ and URC2.

PROOF

[URC1]. Suppose that $\alpha_{i} a \succsim \beta_{i} b$ and $\gamma_{j} c \succsim \delta_{j} d$. Using model (UM) we have:

$$
F\left([p(\alpha, \beta)]_{i},\left[p\left(a_{k}, b_{k}\right)\right]_{k \neq i}\right) \geq 0 \text { and } F\left([p(\gamma, \delta)]_{j},\left[p\left(c_{\ell}, d_{\ell}\right)\right]_{\ell \neq j}\right) \geq 0
$$

with $[\cdot]_{i}$ denoting the $i$ th argument of $F$. If $p(\alpha, \beta) \geq p(\gamma, \delta)$ then using the nondecreasingness of $F$, we have $F\left([p(\alpha, \beta)]_{j},\left[p\left(c_{\ell}, d_{\ell}\right)\right]_{\ell \neq j}\right) \geq 0$ so that $\alpha_{j} c \succsim \beta_{j} d$. If $p(\alpha, \beta)<p(\gamma, \delta)$ we have $F\left([p(\gamma, \delta)]_{i},\left[p\left(a_{k}, b_{k}\right)\right]_{k \neq i}\right) \geq 0$ so that $\gamma_{i} a \succsim \delta_{i} b$. Hence URC1 holds.

[URC2]. Similarly, suppose that $\alpha_{i} a \succsim \beta_{i} b$ and $\beta_{j} c \succsim \alpha_{j} d$. We thus have:

$$
F\left([p(\alpha, \beta)]_{i},\left[p\left(a_{k}, b_{k}\right)\right]_{k \neq i}\right) \geq 0 \text { and } F\left([p(\beta, \alpha)]_{j},\left[p\left(c_{\ell}, d_{\ell}\right)\right]_{\ell \neq j}\right) \geq 0 .
$$

If $p(\alpha, \beta) \geq p(\gamma, \delta)$, the skew symmetry of $p$ implies $p(\delta, \gamma) \geq p(\beta, \alpha)$. Using the nondecreasingness of $F$, we have $F\left([p(\delta, \gamma)]_{j},\left[p\left(c_{\ell}, d_{\ell}\right)\right]_{\ell \neq j}\right) \geq 0$, so that $\delta_{j} c \succsim \gamma_{j} d$. Similarly, if $p(\alpha, \beta)<p(\gamma, \delta)$, we have, using the nondecreasingness of $F, F\left([p(\gamma, \delta)]_{i},\left[p\left(a_{k}, b_{k}\right)\right]_{k \neq i}\right)$ $\geq 0$ so that $\gamma_{i} a \succsim \delta_{i} b$. Hence URC2 holds. 
It turns out that conditions URC1 and URC2 allow to completely characterize model (UM) when $\Gamma / \sim^{* *}$ is finite or countably infinite.

\section{Theorem 1}

Let $\succsim$ be a binary relation on $\mathcal{A}$. If $\Gamma / \sim^{* *}$ is finite or countably infinite, then $\succsim$ has a representation (UM) iff it is reflexive and satisfies URC1 and URC2.

PROOF

Necessity follows from lemmas 2 and 5. We establish sufficiency.

Since URC1 and URC2 hold, we know from lemma 4 that $\succsim^{* *}$ is complete so that it is a weak order. This implies that $\succsim^{*}$ is a weak order. Since $\Gamma / \sim^{* *}$ is finite or countably infinite, it is clear that $\Gamma / \sim^{*}$ is finite or countably infinite. Therefore, there is a real-valued function $q$ on $\Gamma^{2}$ such that, for all $\alpha, \beta, \gamma, \delta \in \Gamma,(\alpha, \beta) \succsim^{*}(\gamma, \delta) \Leftrightarrow q(\alpha, \beta) \geq q(\gamma, \delta)$. Given a particular numerical representation $q$ of $\succsim^{*}$, let $p(\alpha, \beta)=q(\alpha, \beta)-q(\beta, \alpha)$. It is obvious that $p$ is skew symmetric and represents $\succsim^{* *}$.

Define $F$ as follows:

$$
F\left(p\left(a_{1}, b_{1}\right), p\left(a_{2}, b_{2}\right), \ldots, p\left(a_{n}, b_{n}\right)\right)=\left\{\begin{array}{cl}
\exp \left(\sum_{i=1}^{n} p\left(a_{i}, b_{i}\right)\right) & \text { if } a \succsim b \\
-\exp \left(-\sum_{i=1}^{n} p\left(a_{i}, b_{i}\right)\right) & \text { otherwise }
\end{array}\right.
$$

The well-definedness of $F$ follows from (4). To show that $F$ is nondecreasing, suppose that $p(\alpha, \beta) \geq p(\gamma, \delta)$, i.e. that $(\alpha, \beta) \succsim^{* *}(\gamma, \delta)$. If $\gamma_{i} a \succsim \delta_{i} b$, we know from (3) that $\alpha_{i} a \succsim \beta_{i} b$ and the conclusion follows from the definition of $F$. If $\operatorname{Not}\left[\gamma_{i} a \succsim \delta_{i} b\right]$, we have either $\operatorname{Not}\left[\alpha_{i} a \succsim \beta_{i} b\right]$ or $\alpha_{i} a \succsim \beta_{i} b$. In either case, the conclusion follows from the definition of $F$. Since $\succsim$ is reflexive, we have $F(0) \geq 0$, as required. This completes the proof.

\section{Remark 1}

Following Bouyssou and Pirlot (2002), it is not difficult to extend theorem 1 to sets of arbitrary cardinality adding a, necessary, condition implying that the weak order $\succsim^{*}$ (and, hence, $\succsim^{* *}$ ) has a numerical representation. This will not be useful here and we leave the details to the interested reader.

We refer to Bouyssou and Pirlot (2002) for an analysis of the, obviously quite weak, uniqueness properties of the numerical representation of model (UM). Observe that, if $\succsim$ has a representation in model (UM), we must have that:

$$
(\alpha, \beta) \succ^{* *}(\gamma, \delta) \Rightarrow p(\alpha, \beta)>p(\gamma, \delta) .
$$

Hence, the number of distinct values taken by $p$ in a representation in model (UM) is an upper bound of the number of distinct equivalence classes of $\succsim^{* *}$.

\section{Remark 2}

Following the analysis in Bouyssou and Pirlot (2002), it is not difficult to analyze variants of model (UM). For instance, when $\Gamma$ is finite or countably infinite: 
- the weakening of model (UM) obtained considering a function $p$ that may not be skew symmetric but is such that $p(\alpha, \alpha)=0$, for all $\alpha \in \Gamma$, is equivalent to supposing that $\succsim$ is reflexive, independent and satisfies URC1,

- the weakening of model (UM) obtained considering a function $F$ that may not be nondecreasing is equivalent to supposing that $\succsim$ is reflexive and independent,

- the strengthening of model (UM) obtained considering a function $F$ that is odd $(F(\mathbf{x})=-F(\mathbf{x}))$ is equivalent to supposing that $\succsim$ is complete and satisfies URC1 and URC2.

In Bouyssou and Pirlot (2004c), we study the strengthening of model (UM) obtained requiring that $F$ that is odd and strictly increasing in each of its arguments. In the finite or countably infinite case, this model is shown to be characterized by the completeness of $\succsim$ and the "Cardinal Coordinate Independence" condition introduced in Wakker (1984, $1988,1989)$ in order to derive the SEU model. This condition implies both URC1 and URC2 for complete relations.

All the above results are easily generalized to cover the case of an arbitrary set of consequences adding appropriate conditions guaranteeing that ${ }^{*}$ has a numerical representation (on these conditions, see Fishburn, 1970; Krantz et al., 1971)

\section{A new characterization of $L D$ relations}

We have analyzed in Bouyssou and Pirlot (2004c) the relations between model (UM) and models (SEU) and (SSA). We show here what has to be added to the conditions of theorem 1 in order to characterize LD relations. The basic intuition behind this analysis is quite simple. Consider a binary relation $\succsim$ that has a representation in model (UM) in which the function $p$ takes at most three distinct values, i.e. a positive value, a null value and a negative value. In such a case, it is tempting to define the relation $\mathcal{S}$ letting $\alpha \mathcal{P} \beta \Leftrightarrow p(\alpha, \beta)>0$ and $\alpha \mathcal{J} \beta \Leftrightarrow p(\alpha, \beta)=0$. Since $p$ takes only three distinct values, the relation $\mathcal{S}$ summarizes without any loss the information contained in the skew symmetric function $p$. This brings us quite close to a LD relation. We formalize this intuition below. This will require the introduction of conditions that will limit the number of equivalence classes of $\sim^{*}$ and, therefore, $\sim^{* *}$. 


\section{Definition 4 (Conditions UM1 and UM2)}

Let $\succsim$ be a binary relation on a set $\mathcal{A}$. This relation is said to satisfy:

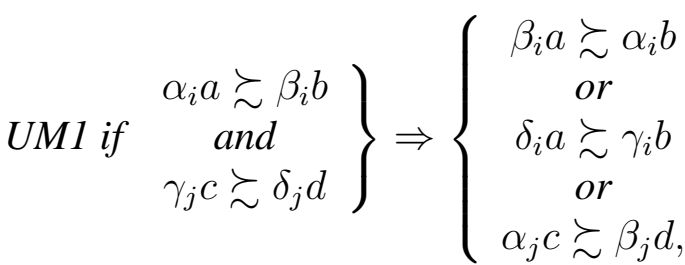

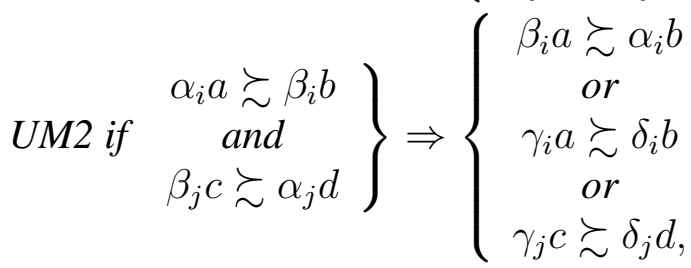

for all $i, j \in N$, all $a, b, c, d \in \mathcal{A}$ and all $\alpha, \beta, \gamma, \delta \in \Gamma$.

In order to analyze these two conditions, it will be useful to introduce the following two conditions:

$$
\begin{aligned}
& \left.\begin{array}{c}
\alpha_{i} a \succsim \beta_{i} b \\
\text { and } \\
\gamma_{j} c \succsim \delta_{j} d
\end{array}\right\} \Rightarrow\left\{\begin{array}{c}
\beta_{i} a \succsim \alpha_{i} b \\
\text { or } \\
\alpha_{j} c \succsim \beta_{j} d,
\end{array}\right. \\
& \left.\begin{array}{c}
\alpha_{i} a \succsim \beta_{i} b \\
\text { and } \\
\beta_{j} c \succsim \alpha_{j} d
\end{array}\right\} \Rightarrow\left\{\begin{array}{c}
\beta_{i} a \underset{ }{\succsim} \alpha_{i} b \\
\text { or } \\
\gamma_{j} c \succsim \delta_{j} d,
\end{array}\right.
\end{aligned}
$$

for all $i, j \in N$, all $a, b, c, d \in \mathcal{A}$ and all $\alpha, \beta, \gamma, \delta \in \Gamma$. Condition (6) has a simple interpretation. Suppose that $\alpha_{i} a \succsim \beta_{i} b$ and $\operatorname{Not}\left[\beta_{i} a \succsim \alpha_{i} b\right]$. This is the sign that the preference difference between $\alpha$ and $\beta$ is strictly larger than the preference difference between $\beta$ and $\alpha$. Because with LD relations there can be only three types of preference differences (positive, null and negative) and preference differences are compared in a reversible way, this implies that the preference difference between $\alpha$ and $\beta$ must be at least as large as any other preference difference. In particular, if $\gamma_{j} c \succsim \delta_{j} d$, it must follow that $\alpha_{j} c \succsim \beta_{j} d$. This is what condition (6) implies. Condition (7) has an obvious dual interpretation: if a difference is strictly smaller than its opposite then any other preference must be at least as large as this difference. Conditions UM1 and UM2 are respectively deduced from (6) and (7) by adding a conclusion to these conditions. This additional conclusion ensures that these new conditions are independent from URC1 and URC2. This is formalized below.

\section{Lemma 6}
1. (6) $\Leftrightarrow\left[\operatorname{Not}\left[(\beta, \alpha) \succsim^{*}(\alpha, \beta)\right] \Rightarrow(\alpha, \beta) \succsim^{*}(\gamma, \delta)\right]$,
2. (7) $\Leftrightarrow\left[N o t\left[(\beta, \alpha) \succsim^{*}(\alpha, \beta)\right] \Rightarrow(\gamma, \delta) \succsim^{*}(\beta, \alpha)\right]$, 
3. (6) $\Rightarrow U M 1$,

4. (7) $\Rightarrow U M 2$,

5. $U R C 2$ and $U M 1 \Rightarrow(6)$,

6. $U R C 1$ and $U M 2 \Rightarrow(7)$,

7. $\left[U R C 1, U R C 2, U M 1\right.$ and UM2] $\Rightarrow\left[\succsim^{* *}\right.$ is a weak order having at most three equivalence classes].

8. In the class of reflexive relations, URC1, URC2, UM1 and UM2 are independent conditions.

PROOF

Part 1 . We clearly have $\operatorname{Not}[(6)] \Leftrightarrow\left[\operatorname{Not}\left[(\beta, \alpha) \succsim^{*}(\alpha, \beta)\right]\right.$ and $\left.\operatorname{Not}\left[(\alpha, \beta) \succsim^{*}(\gamma, \delta)\right]\right]$. The proof of part 2 is similar. Parts 3 and 4 are obvious since UM1 (resp. UM2) amounts to adding a possible conclusion to (6) (resp. (7)).

Part 5. Suppose that $\alpha_{i} a \succsim \beta_{i} b$ and $\gamma_{j} c \succsim \delta_{j} d$. If $\operatorname{Not}\left[\delta_{j} a \succsim \gamma_{j} b\right]$, UM1 implies $\beta_{i} a \succsim \alpha_{i} b$ or $\alpha_{j} c \succsim \beta_{j} d$. Suppose now that $\delta_{j} a \succsim \gamma_{j} b$. Using URC2 $\delta_{i} a \succsim \gamma_{i} b$ and $\gamma_{j} a \succsim \delta_{j} b$ imply $\beta_{i} a \succsim \alpha_{i} b$ or $\alpha_{j} a \succsim \beta_{j} b$. Hence, (6) holds.

Part 6. Suppose that $\alpha_{i} a \succsim \beta_{i} b$ and $\beta_{j} c \succsim \alpha_{j} d$. If $\operatorname{Not}\left[\gamma_{i} a \succsim \delta_{i} b\right]$, UM2 implies $\beta_{i} a \succsim \alpha_{i} b$ or $\gamma_{j} c \succsim \delta_{j} d$. Suppose now that $\gamma_{i} a \succsim \delta_{i} b$. Using URC1 $\gamma_{i} a \succsim \delta_{i} b$ and $\beta_{j} c \succsim \alpha_{j} d$ imply $\beta_{i} a \succsim \alpha_{i} b$ or $\gamma_{j} c \succsim \delta_{j} d$. Hence, (7) holds.

Part 7. Since URC1 and URC2 hold, we know that $\succsim^{* *}$ is complete. Since $\succsim^{* *}$ is reversible, the conclusion will be false iff there are $\alpha, \beta, \gamma, \delta \in \Gamma$ such that $(\alpha, \beta) \succ^{* *}$ $(\gamma, \delta) \succ^{* *}(\alpha, \alpha)$.

1. Suppose that $(\alpha, \beta) \succ^{*}(\gamma, \delta)$ and $(\gamma, \delta) \succ^{*}(\alpha, \alpha)$. Using URC2, we know that $(\alpha, \alpha) \succsim^{*}(\delta, \gamma)$. Using the transitivity of $\succsim^{*}$ we have $(\gamma, \delta) \succ^{*}(\delta, \gamma)$. Since $(\alpha, \beta) \succ^{*}(\gamma, \delta)$, this contradicts (6).

2. Suppose that $(\alpha, \beta) \succ^{*}(\gamma, \delta)$ and $(\alpha, \alpha) \succ^{*}(\delta, \gamma)$. Using URC2, we know that $(\gamma, \delta) \succsim^{*}(\alpha, \alpha)$. Using the transitivity of $\succsim^{*}$ we have $(\gamma, \delta) \succ^{*}(\delta, \gamma)$. Since $(\alpha, \beta) \succ^{*}(\gamma, \delta)$, this contradicts (6).

3. Suppose that $(\delta, \gamma) \succ^{*}(\beta, \alpha)$ and $(\gamma, \delta) \succ^{*}(\alpha, \alpha)$. Using URC2, we know that $(\alpha, \alpha) \succsim^{*}(\delta, \gamma)$ so that $(\gamma, \delta) \succ^{*}(\delta, \gamma)$. Since $(\delta, \gamma) \succ^{*}(\beta, \alpha)$, this contradicts (7).

4. Suppose that $(\delta, \gamma) \succ^{*}(\beta, \alpha)$ and $(\alpha, \alpha) \succ^{*}(\delta, \gamma)$. Using URC2 we have $(\gamma, \delta) \succsim^{*}$ $(\alpha, \alpha)$ so that $(\gamma, \delta) \succ^{*}(\delta, \gamma)$. Since $(\delta, \gamma) \succ^{*}(\beta, \alpha)$, this contradicts (7).

Part 8: see examples 4, 5, 6 and 7 in appendix. 
In view of the above lemma, conditions UM1 and UM2 seem to adequately capture the ordinal character of the aggregation at work in a LD relation within the framework of model (UM). Indeed, the following lemma shows that all LD relations satisfy UM1 and UM2 while having a representation in model (UM).

\section{Lemma 7}

Let $\succsim$ be a binary relation on $\mathcal{A}$. If $\succsim$ is a $L D$ relation then,
1. ¿ satisfies URC1 and URC2,
2. $\succsim$ satisfies $U M 1$ and $U M 2$.

\section{PROOF}

Let $\langle\unrhd, \mathcal{S}\rangle$ be the representation of $\succsim$.

Part 1. Let us show that URC1 holds, i.e. that $\alpha_{i} a \succsim \beta_{i} b$ and $\gamma_{j} c \succsim \delta_{j} d$ imply $\gamma_{i} a \succsim \delta_{i} b$ or $\alpha_{j} c \succsim \beta_{j} d$.

There are 9 cases to envisage:

\begin{tabular}{cccc} 
& $\gamma \mathcal{P} \delta$ & $\gamma \mathcal{J} \delta$ & $\delta \mathcal{P} \gamma$ \\
\hline$\alpha \mathcal{P} \beta$ & $(i)$ & $($ ii $)$ & $($ iii $)$ \\
$\alpha \mathcal{J} \beta$ & $($ iv $)$ & $(v)$ & $(v i)$ \\
$\beta \mathcal{P} \alpha$ & $($ vii $)$ & $($ viii $)$ & $($ ix $)$
\end{tabular}

Cases $(i),(v)$ and (ix) clearly follow from (2). All other cases easily follow from (2) and the monotonicity of $\unrhd$. The proof for URC2 is similar.

Part 2. Let us show that UM1 holds, i.e. that $\alpha_{i} a \succsim \beta_{i} b$ and $\gamma_{j} c \succsim \delta_{j} d$ imply $\beta_{i} a \succsim \alpha_{i} b$ or $\gamma_{i} a \succsim \delta_{i} b$ or $\alpha_{j} c \succsim \beta_{j} d$.

If $\alpha \mathcal{P} \beta$ then, using (2) and the monotonicity of $\unrhd, \gamma_{j} c \succsim \delta_{j} d$ implies $\alpha_{j} c \succsim \beta_{j} d$. If $\beta \mathcal{P} \alpha$ then, using (2) and the monotonicity of $\unrhd, \alpha_{i} a \succsim \beta_{i} b$ implies $\beta_{i} a \succsim \alpha_{i} b$. If $\alpha$ J $\beta$, then $\beta$ J $\alpha$ so that, using (2), $\alpha_{i} a \succsim \beta_{i} b$ implies $\beta_{i} a \succsim \alpha_{i} b$. The proof for UM2 is similar.

We are now in position to present the main result of this section.

\section{Theorem 2}

Let $\succsim$ be a binary relation on $\mathcal{A}$. Then $\succsim$ is a LD relation iff it is reflexive and satisfies URC1, URC2, UM1 and UM2.

PROOF

Necessity follows from lemma 7 and the definition of a LD relation. We show that if $\succsim$ satisfies URC1 and URC2 and is such that $\sim^{* *}$ has at most three distinct equivalence classes then $\succsim$ is a LD relation. In view of lemma 6 , this will establish sufficiency. 
Define $\mathcal{S}$ letting, for all $\alpha, \beta \in \Gamma, \alpha \mathcal{S} \beta \Leftrightarrow(\alpha, \beta) \succsim^{* *}(\beta, \beta)$. By hypothesis, we know that $\succsim^{* *}$ is complete and $\succsim$ is independent. It easily follows that $\mathcal{S}$ is complete.

The relation $\succsim^{*}$ being complete, the influence of $i \in N$ implies that there are $\gamma, \delta, \alpha, \beta \in$ $\Gamma$ such that $(\alpha, \beta) \succ^{*}(\gamma, \delta)$. Since $\succsim^{* *}$ is complete, this implies $(\alpha, \beta) \succ^{* *}(\gamma, \delta)$. If $(\alpha, \beta) \succ^{* *}(\beta, \beta)$ then $\alpha \mathcal{P} \beta$. If not, then $(\beta, \beta) \succsim^{* *}(\alpha, \beta)$ so that $(\beta, \beta) \succ^{* *}(\gamma, \delta)$ and, using the reversibility of $\succsim^{* *}$ and the independence of $\succsim, \delta \mathcal{P} \gamma$. This shows that $\mathcal{P}$ is not empty. This implies that $\succsim^{* *}$ has exactly three distinct equivalence classes, since $\alpha \mathcal{P} \beta \Leftrightarrow(\alpha, \beta) \succ^{* *}(\beta, \beta) \Leftrightarrow(\beta, \beta) \succ^{* *}(\beta, \alpha)$. Therefore, $\alpha \mathcal{P} \beta$ iff $(\alpha, \beta)$ belongs to the first equivalence class of $\succsim^{* *}$ and $(\beta, \alpha)$ to its last equivalence class. Consider any two subsets $A, B \subseteq N$ such that $A \cup B=N$ and let:

$$
A \unrhd B \Leftrightarrow[a \succsim b, \text { for some } a, b \in \mathcal{A} \text { such that } \mathcal{S}(a, b)=A \text { and } \mathcal{S}(b, a)=B] \text {. }
$$

If $a \succsim b$ then, by construction, we have $\mathcal{S}(a, b) \unrhd \mathcal{S}(b, a)$. Suppose now that $\mathcal{S}(a, b) \unrhd$ $\mathcal{S}(b, a)$, so that there are $c, b \in \mathcal{A}$ such that $c \succsim d$ and $\left(c_{i}, d_{i}\right) \sim^{* *}\left(a_{i}, b_{i}\right)$, for all $i \in N$. Using (4), we have $a \succsim b$. Hence (2) holds. The monotonicity of $\unrhd$ easily follows from (3). This completes the proof.

We have therefore obtained a complete characterization of LD relation within the general framework of model (UM). Conditions UM1 and UM2 implying that $\succsim^{* *}$ has at most three distinct equivalence classes appear as the main distinctive characteristic of LD relations. Clearly a binary relation $\succsim$ having a representation in models (SEU) or (SSA) will, in general, have a much richer relation $\succsim^{* *}$.

\section{Discussion and extensions}

The purpose of this paper was twofold. We have first introduced a general axiomatic framework for decision under uncertainty that contains both the SEU and the LD models as particular cases. This model, while tolerating intransitive and/or incomplete preferences, has a simple and intuitive interpretation in terms of preference differences. It is nontrivial unlike, e.g., the general model introduced in Chu and Halpern (2003). We showed that it can be characterized using simple conditions, while avoiding the use of any unnecessary structural assumptions. The second aim of this paper was to put our general framework to work, using it to propose an alternative characterization of the preference relations that can be obtained using the likely dominance rule. This characterization has emphasized the main specific feature of LD relations, i.e. the fact that they use a very poor information concerning preference differences admitting only "positive", "null" and "negative" differences. 


\subsection{Comparison with Fargier and Perny (1999) and Dubois et al. (2003a)}

We compare below our characterization of LD relations with the one proposed in Fargier and Perny (1999); closely related results are found in Dubois et al. (2003a, 2002). Their characterization is based on a condition called "qualitative independence" (and later called "ordinal invariance" in Dubois et al. (2003a, 2002)) that is a slight variant (using a reflexive relation instead of an asymmetric one) of the "noncompensation" condition introduced in Fishburn $(1975,1976,1978)$ which, in turn, is a "single profile" analogue of the independence condition used in Arrow's theorem (see Sen, 1986).

Since our definition of LD relations differs from the one used in Fargier and Perny (1999) (they do not impose that $\unrhd$ is necessarily monotonic w.r.t. inclusion) we reformulate their result below. For any $a, b \in \mathcal{A}$, let $R(a, b)=\left\{i \in N: a_{i} \succsim_{\Gamma} b_{i}\right\}$.

\section{Definition 5}

Let $\succsim$ be a binary relation on $\mathcal{A}$. This relation is said to satisfy monotonic qualitative independence $(M Q I)$ if,

$$
\left.\begin{array}{l}
R(a, b) \supseteq R(c, d) \\
\text { and } \\
R(b, a) \subseteq R(d, c)
\end{array}\right\} \Rightarrow[c \succsim d \Rightarrow a \succsim b],
$$

for all $a, b, c, d \in \mathcal{A}$.

Condition MQI is strengthens the "qualitative independence" condition used in Fargier and Perny (1999) (this condition is obtained replacing inclusions by equalities in the expression of MQI; as observed in Dubois et al. (2003a, 2002), it is also possible to use instead of MQI the original qualitative independence condition together with a condition imposing that $\succsim$ is monotonic w.r.t. $\succsim_{\Gamma}$ ) to include an idea of monotonicity. Condition MQI is a "single profile" analogue of the NIM (i.e., Neutrality, Independence, Monotonicity) condition that is classical in Social Choice Theory (see Sen, 1986, p. 1086).

As shown below, in what is an adaptation of Fargier and Perny (1999, proposition 5), this condition allows for a very simple characterization of LD relations.

\section{Proposition 1}

Let $\succsim$ be a binary relation on $\mathcal{A}$. The relation $\succsim$ is a $L D$ relation iff

- $\succsim$ is reflexive,

- ${ }_{\Gamma}$ is complete,

- $\succsim$ satisfies $M Q I$. 


\section{PROOF}

Necessity. Reflexivity holds by definition of a LD relation. That $\succsim_{\Gamma}$ must be complete follows from part 3 of lemma 2. The necessity of MQI follows from (2), using the monotonicity of $\unrhd$ and part 7 of lemma 1 .

Sufficiency. Let $\mathcal{S}=\succsim_{\Gamma}$. By hypothesis, $\mathcal{S}$ is complete. If $\succ_{\Gamma}$ is empty, we have $R(a, b)=N$ for all $a, b \in \mathcal{A}$. Using the reflexivity of $\succsim$ and $M Q I$ this implies that $a \succsim b$, for all $a, b \in \mathcal{A}$ and, hence, that all states $i \in N$ are degenerate, contrary to our hypothesis. Hence $\succ_{\Gamma}=\mathcal{P}$ is nonempty.

Let $A, B \subseteq N$ such that $A \cup B=N$. Since $\mathcal{P}$ is nonempty there are $a, b \in \mathcal{A}$ such that $\mathcal{S}(a, b)=A$ and $\mathcal{S}(b, a)=B$. Define $\unrhd$ letting:

$$
A \unrhd B \Leftrightarrow[a \succsim b \text {, for some } a, b \in \mathcal{A} \text { such that } \mathcal{S}(a, b)=A \text { and } \mathcal{S}(b, a)=B] .
$$

If $a \succsim b$ then, by construction, we have $\mathcal{S}(a, b) \unrhd \mathcal{S}(b, a)$. Suppose now that $\mathcal{S}(a, b) \unrhd$ $\mathcal{S}(b, a)$. By construction, there are $c, d \in \mathcal{A}$ such that $c \succsim d$ and $\mathcal{S}(c, d)=A$ and $\mathcal{S}(d, c)=B$. Using MQI, it follows that $a \succsim b$. That $\unrhd$ is monotonic w.r.t. inclusion clearly follows from MQI.

We refer to Dubois et al. (2002); Fargier and Perny (1999) for a thorough analysis of this result, including a careful comparison of the above conditions with the classical ones used in Savage (1954).

Although proposition 1 offers a simple characterization of LD relations, condition MQI appears at the same time quite strong (this will be apparent if one tries to reformulate MQI in terms of $\succsim)$ and wholly specific to LD relations. In our view, the characterization of LD relations within model (UM) proposed above allows to better isolate what appears to be the specific features of LD relations while showing their links with more classical preference relations used in the field of decision under uncertainty.

It should also be stressed that the characterization of LD relations is far from being the only objective of the above-mentioned papers. Rather, their aim is to study the, drastic, consequences of supposing that $\succsim$ is a LD relation and has nice transitivity properties (e.g. $\succ$ being transitive or without circuits). This analysis, that is closely related to Arrow-like theorems in Social Choice Theory (see Campbell and Kelly, 2002; Sen, 1986, for overviews), illuminates the relations between the LD rule, possibility theory and nonmonotonic reasoning. Such an analysis is clearly independent from the path followed to characterize LD relations.

\subsection{Extensions}

As already mentioned, model (UM) is the specialization to the case of decision making under uncertainty of the conjoint measurement models proposed in Bouyssou and Pirlot 
(2002). It is not difficult to see that model (UM) not only allows for intransitive relations $\succsim$ between acts but also for intransitive relation $\succsim_{\Gamma}$ between outcomes. This may be seen as a limitation of model (UM). Indeed, whereas intransitivities are not unlikely when comparing acts (see Fishburn, 1991), one would expect a much more well behaved relation when it turns to comparing outcomes. We show in this section how to extend our results to cover this case. Before doing so, let us stress that it is quite remarkable that any transitivity hypothesis is unnecessary to obtain a complete characterization of LD relations. As forcefully argued in Saari (1998), this seems to be an essential feature of "ordinal" models.

Adapting the analysis in Bouyssou and Pirlot (2004a) to the case of decision under uncertainty, let us first show that it is possible to specialize model (UM) in order introduce a linear arrangement of the elements of $\Gamma$. We consider binary relations $\succsim$ on $\mathcal{A}$ that can be represented as:

$$
a \succsim b \Leftrightarrow F\left(\varphi\left(u\left(a_{1}\right), u\left(b_{1}\right)\right), \ldots, \varphi\left(u\left(a_{n}\right), u\left(b_{n}\right)\right)\right) \geq 0
$$

where $u$ is a real-valued function on $\Gamma, \varphi$ is a real-valued function on $u(\Gamma)^{2}$ that is skew symmetric, nondecreasing in its first argument (and, therefore, nonincreasing in its second argument) and $F$ is a real-valued function on $\prod_{i=1}^{n} \varphi\left(u(\Gamma)^{2}\right)$ being nondecreasing in all its arguments and such that $F(\mathbf{0}) \geq 0$.

Comparing models $\left(\mathrm{UM}^{*}\right)$ and $(\mathrm{UM})$, it is clear that $\left(\mathrm{UM}^{*}\right)$ is the special case of model (UM) in which the function $p$ measuring preference differences between outcomes may be factorised using a function $u$ measuring the "utility" of the outcomes and a skew symmetric function $\varphi$ measuring preference differences between outcomes on the basis of $u$. It is easy to see that model (UM*) implies that $\succsim_{\Gamma}$ is complete and that $\succ_{\Gamma}$ is transitive. The analysis below will, in fact, show that model (UM*) implies that $\succsim_{\Gamma}$ is a semiorder.

The analysis of model $\left(\mathrm{UM}^{*}\right)$ will require the introduction of three new conditions inspired from Bouyssou and Pirlot (2004a).

\section{Definition 6 (Conditions UAC1, UAC2 and UAC3)}

We say that $\succsim$ satisfies:

$$
\begin{aligned}
& \text { UACl if } \left.\begin{array}{c}
\alpha_{i} a \succsim b \\
\text { and } \\
\beta_{j} c \succsim d
\end{array}\right\} \Rightarrow\left\{\begin{array}{c}
\beta_{i} a \succsim b \\
o r \\
\alpha_{j} c \succsim d,
\end{array}\right. \\
& \text { UAC2 if } \left.\begin{array}{c}
a \succsim \alpha_{i} b \\
\text { and } \\
c \succsim \beta_{j} d
\end{array}\right\} \Rightarrow\left\{\begin{array}{c}
a \succsim \beta_{i} b \\
\text { or } \\
c \succsim \alpha_{j} d,
\end{array}\right. \\
& \text { UAC3 if } \left.\begin{array}{c}
a \succsim \alpha_{i} b \\
\text { and } \\
\alpha_{j} c \succsim d
\end{array}\right\} \Rightarrow\left\{\begin{array}{c}
a \succsim \beta_{i} b \\
\text { or } \\
\beta_{j} c \succsim d,
\end{array}\right.
\end{aligned}
$$

for all $a, b, c, d \in \mathcal{A}$, all $i, j \in N$ and all $\alpha, \beta \in \Gamma$. 
Condition UAC1 suggests that the elements of $\Gamma$ can be linearly ordered considering "upward dominance": if $\alpha$ "upward dominates" $\beta$ then $\beta_{i} a \succsim b$ entails $\alpha_{i} a \succsim b$, for all $a, b \in \mathcal{A}$ and all $i \in N$. Condition UAC2 has a similar interpretation considering now "downward dominance". Condition UAC3 ensures that the linear arrangements of the elements of $\Gamma$ obtained considering upward and downward dominance are not incompatible. The study of the impact of these new conditions on model (UM) will require an additional definition borrowed from Doignon et al. (1988).

\section{Definition 7 (Linearity)}

Let $\mathcal{R}$ be a binary relation on a set $X^{2}$. We say that:

- $\mathcal{R}$ is right-linear iff $[\operatorname{Not}[(y, z) \mathcal{R}(x, z)] \Rightarrow(x, w) \mathcal{R}(y, w)]$,

- $\mathcal{R}$ is left-linear iff $[\operatorname{Not}[(z, x) \mathcal{R}(z, y)] \Rightarrow(w, y) \mathcal{R}(w, x)]$,

- $\mathcal{R}$ is strongly linear iff $[\operatorname{Not}[(y, z) \mathcal{R}(x, z)]$ or $\operatorname{Not}[(z, x) \mathcal{R}(z, y)]] \Rightarrow[(x, w) \mathcal{R}$ $(y, w)$ and $(w, y) \mathcal{R}(w, x)]$,

for all $x, y, z, w \in X$.

The impact of our new conditions on the relations $\succsim^{*}$ and $\succsim^{* *}$ comparing preference differences between outcomes are noted below.

\section{Lemma 8}

1. $U A C 1 \Leftrightarrow \succsim^{*}$ is right-linear,

2. $U A C 2 \Leftrightarrow \succsim^{*}$ is left-linear,

3. UAC3 $\Leftrightarrow\left[\left[\operatorname{Not}\left[(\alpha, \gamma) \succsim^{*}(\beta, \gamma)\right]\right.\right.$ for some $\left.\gamma \in \Gamma\right] \Rightarrow\left[(\delta, \alpha) \succsim^{*}(\delta, \beta)\right.$, for all $\delta \in \Gamma]]$

4. $[U A C 1, U A C 2$ and $U A C 3] \Leftrightarrow \succsim^{*}$ is strongly linear $\Leftrightarrow \succsim^{* *}$ is strongly linear.

5. In the class of reflexive relations satisfying URC1 and URC2, UAC1, UAC2 and UAC 3 are independent conditions.

\section{PROOF}

Part 1. ${ }^{*}$ is not right-linear iff for some $\alpha, \beta, \gamma, \delta \in \Gamma$, we have $\operatorname{Not}\left[(\gamma, \beta) \succsim^{*}(\alpha, \beta)\right]$ and $\operatorname{Not}\left[(\alpha, \delta) \succsim^{*}(\gamma, \delta)\right]$. This equivalent to

$$
\begin{aligned}
& {\left[\alpha_{i} a \succsim \beta_{i} b\right] \text { and } \operatorname{Not}\left[\gamma_{i} a \succsim \beta_{i} b\right] \text { and }} \\
& {\left[\gamma_{j} c \succsim \delta_{j} d\right] \text { and } \operatorname{Not}\left[\alpha_{j} c \succsim \delta_{j} d\right],}
\end{aligned}
$$

for some $a, b, c, d \in \mathcal{A}$ and some $i, j \in N$. This is exactly Not[UAC1]. Parts 2 and 3 are established similarly. 
Part 4. The first equivalence is immediate from parts 1 to 3 . The second equivalence directly results from the definitions of $\succsim^{*}$ and $\succsim^{* *}$.

Part 5: see examples 8, 9 and 10 in appendix.

We summarize some useful consequences of model (UM*) in the following:

\section{Lemma 9}

Let $\succsim$ be a binary relation on $\mathcal{A}$. If $\succsim$ has a representation in (UM*) then:

1. it satisfies $U R C 1$ and URC2,

2. it satisfies UAC1, UAC2 and UAC3,

3. the binary relation $T$ on $\Gamma$ defined by $\alpha T \beta \Leftrightarrow(\alpha, \beta) \succsim^{* *}(\alpha, \alpha)$ is a semiorder.

\section{PROOF}

Part 1 follows from the definition of model $\left(\mathrm{UM}^{*}\right)$ and theorem 1.

Part 2. Suppose that $\alpha_{i} a \succsim b$ and $\beta_{j} c \succsim d$. This implies, abusing notation,

$$
\begin{aligned}
& F\left(\left[\varphi\left(u(\alpha), u\left(b_{i}\right)\right)\right]_{i},\left[\varphi\left(u\left(a_{k}\right), u\left(b_{k}\right)\right)\right]_{k \neq i}\right) \geq 0 \text { and } \\
& F\left(\left[\varphi\left(u(\beta), u\left(d_{j}\right)\right)\right]_{j},\left[\varphi\left(u\left(c_{\ell}\right), u\left(d_{\ell}\right)\right)\right]_{\ell \neq j}\right) \geq 0 .
\end{aligned}
$$

If $u(\beta)<u(\alpha)$, since $\varphi$ is nondecreasing in its first argument and $F$ is nondecreasing in all its arguments, we obtain

$$
F\left(\left[\varphi\left(u(\alpha), u\left(d_{j}\right)\right)\right]_{j},\left[\varphi\left(u\left(c_{\ell}\right), u\left(d_{\ell}\right)\right)\right]_{\ell \neq j}\right) \geq 0,
$$

so that $\alpha_{j} c \succsim d$. If $u(\beta) \geq u(\alpha)$, since $\varphi$ is nondecreasing in its first argument and $F$ is nondecreasing in all its arguments, we obtain

$$
F\left(\left[\varphi\left(u(\beta), u\left(b_{i}\right)\right)\right]_{i},\left[\varphi\left(u\left(a_{k}\right), u\left(b_{k}\right)\right)\right]_{k \neq i}\right) \geq 0
$$

so that $\beta_{i} a \succsim b$. Hence, UAC1 holds. The proof is similar for UAC2 and UAC3.

Part 3. Since URC1 and URC2 hold, we know from lemma 4 that $\succsim^{* *}$ is complete. It is reversible by construction. From lemma 8 , we know that $\succsim^{* *}$ is strongly linear. From the proof of theorem 2, we know that $T$ is complete. It remains to show that it is Ferrers and semi-transitive.

[Ferrers]. Suppose that $\alpha T \beta$ and $\gamma T \delta$ so that $(\alpha, \beta) \succsim^{* *}(\beta, \beta)$ and $(\gamma, \delta) \succsim^{* *}$ $(\delta, \delta)$. In contradiction with the thesis, suppose that $\operatorname{Not}[\alpha T \delta]$ and $\operatorname{Not}[\gamma T \beta]$ so that $(\delta, \delta) \succ^{* *}(\alpha, \delta)$ and $(\beta, \beta) \succ^{* *}(\gamma, \beta)$. Using the fact that $\succsim^{* *}$ is a weak order, this implies $(\alpha, \beta) \succ^{* *}(\gamma, \beta)$ and $(\gamma, \delta) \succ^{* *}(\alpha, \delta)$. This violates the strong linearity of $\succsim^{* *}$. 
[Semi-transitivity]. Suppose that $\alpha$ T $\beta$ and $\beta T \gamma$ so that $(\alpha, \beta) \succsim^{* *}(\beta, \beta)$ and $(\beta, \gamma) \succsim^{* *}(\gamma, \gamma)$. In contradiction with the thesis, suppose that $\operatorname{Not}[\alpha T \delta]$ and $\operatorname{Not}[\delta T \gamma]$ so that $(\delta, \delta) \succ^{* *}(\alpha, \delta)$ and $(\gamma, \gamma) \succ^{* *}(\delta, \gamma)$. Using the fact that $\succsim^{* *}$ is a reversible weak order, we obtain $(\alpha, \beta) \succ^{* *}(\alpha, \delta)$ and $(\beta, \gamma) \succ^{* *}(\delta, \gamma)$. This violates the strong linearity of $\succsim^{* *}$. Hence, $T$ is semi-transitive.

The conditions introduced so far allow us to characterize model (UM*) when $\Gamma$ and, hence, $\mathcal{A}$, is at most denumerable.

\section{Theorem 3}

Suppose that $\Gamma$ is finite or countably infinite and let $\succsim$ be a binary relation on $\mathcal{A}$. Then $\succsim$ has a representation (UM*) iff it is reflexive and satisfies URC1, URC2, UAC1, UAC2 and UAC3.

\section{PROOF}

Necessity results from lemmas 2, 5 and 9 . The proof of sufficiency rests on the following claim proved in Bouyssou and Pirlot (2004a, Proposition 2).

Claim Let $\mathcal{R}$ be a weak order on a finite or countably infinite set $X^{2}$. There is a realvalued function $u$ on $X$ and a real-valued function $\varphi$ on $u(X)^{2}$ being nondecreasing in its first argument and nonincreasing in its second argument, such that, for all $x, y, z, w \in X$,

$$
(x, y) \mathcal{R}(z, w) \Leftrightarrow \varphi(u(x), u(y)) \geq \varphi(u(z), u(w))
$$

iff $\mathcal{R}$ is strongly linear. In addition, the function $\varphi$ can be chosen to be skew-symmetric iff $\mathcal{R}$ is reversible.

Sufficiency follows from combining theorem 1 with lemma 8 and the above claim.

\section{Remark 3}

The above result can be extended without much difficulty to sets of arbitrary cardinality. Note however that, contrary to theorem 1 , theorem 3 is only stated here for finite or countably infinite sets $\mathcal{A}$. This is no mistake. In fact, as shown in Fishburn (1973, Theorem A(ii)), it may well happen that $\mathcal{R}$ is a strongly linear weak order on $X^{2}$, that the set of equivalence classes induced by $\mathcal{R}$ is finite or countably infinite while the above claim fails.

We now use the framework of model ( $\mathrm{UM}^{*}$ ) to analyze LD relations in which $\mathcal{S}$ is a semiorder. Let us first show that all such relations have a representation in model (UM*).

\section{Lemma 10}

Let $\succsim$ be a binary relation on $\mathcal{A}$. If $\succsim$ is a $L D$ relation with a representation $\langle\unrhd, \mathcal{S}\rangle$ in which $\mathcal{S}$ is a semiorder then $\succsim$ satisfies UAC1, UAC2 and UAC3. 


\section{PROOF}

[UAC1]. Suppose that $\alpha_{i} a \succsim b$ and $\beta_{j} c \succsim d$. We want to show that either $\beta_{i} a \succsim b$ or $\alpha_{j} c \succsim d$.

If $b_{i} \mathcal{P} \alpha$ or $d_{j} \mathcal{P} \beta$, the conclusion follows from the monotonicity of $\unrhd$.

If $\alpha \mathcal{P} b_{i}$ and $\beta \mathcal{P} d_{j}$, we have, using the fact that $\mathcal{P}$ is Ferrers, $\alpha \mathcal{P} d_{j}$ or $\beta \mathcal{P} b_{i}$. In either case the desired conclusion follows using the fact that $\succsim$ is a LD relation.

This leaves three exclusive cases: $\left[\alpha \mathcal{J} b_{i}\right.$ and $\left.\beta \mathcal{P} d_{j}\right]$ or $\left[\alpha \mathcal{P} b_{i}\right.$ and $\left.\beta \mathcal{J} d_{j}\right]$, or $\left[\alpha \mathcal{J} b_{i}\right.$ and $\beta \mathcal{J} d_{j}$. . Using Ferrers, either case implies $\alpha \mathcal{S} d_{j}$ or $\beta \mathcal{S} b_{i}$. If either $\alpha \mathcal{P} d_{j}$ or $\beta \mathcal{P} b_{i}$, the desired conclusion follows from monotonicity. Suppose therefore that $\alpha \mathcal{J} d_{j}$ and $\beta \mathcal{J} b_{i}$. Since we have either $\alpha \mathcal{J} b_{i}$ or $\beta \mathcal{J} d_{j}$, the conclusion follows using the fact that $\succsim$ is a LD relation.

Hence UAC1 holds. The proof for UAC2 is similar, using Ferrers.

[UAC3]. Suppose that $a \succsim \alpha_{i} b$ and $\alpha_{j} c \succsim d$. We want to show that either $a \succsim \beta_{i} b$ or $\beta_{j} c \succsim d$.

If either $\alpha \mathcal{P} a_{i}$ or $d_{j} \mathcal{P} \alpha$, the conclusion follows from monotonicity.

If $a_{i} \mathcal{P} \alpha$ and $\alpha \mathcal{P} d_{j}$, then semi-transitivity implies $a_{i} \mathcal{P} \beta$ or $\beta \mathcal{P} d_{j}$. In either case, the conclusion follows from monotonicity.

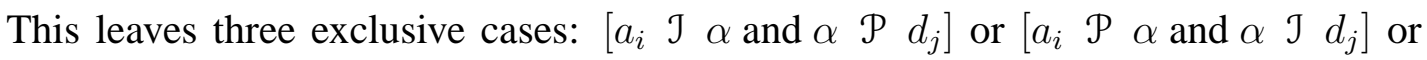
$\left[a_{i} \mathcal{J} \alpha\right.$ and $\left.\alpha \mathcal{J} d_{j}\right]$. In either case, semi-transitivity implies $a_{i} \mathcal{S} \beta$ or $\beta \mathcal{S} d_{j}$. If either $a_{i} \mathcal{P} \beta$ or $\beta \mathcal{P} d_{j}$. the desired conclusion follows from monotonicity. Suppose therefore that $a_{i} \mathcal{J} \beta$ or $\beta \mathcal{J} d_{j}$. Since in each of the remaining cases we have either $a_{i} \mathcal{J} \alpha$ or $\alpha \mathcal{J} d_{j}$, the conclusion follows because $\succsim$ is a LD relation.

Although lemma 8 shows that in the class of reflexive binary relations satisfying URC1 and URC2, UAC1, UAC2 and UAC3 are independent conditions, the situation is more delicate when we bring conditions UM1 and UM2 into the picture since they impose strong requirements on $\succsim^{*}$ and $\succsim^{* *}$. We have:

\section{Lemma 11}

1. Let $\succsim$ be a reflexive binary relation on $\mathcal{A}$ satisfying $U R C 1, U R C 2, U M 1$ and UM2. Then $\succsim$ satisfies UAC1 iff it satisfies UAC2.

2. In the class of reflexive binary relations satisfying URC1, URC2, UM1 and UM2, conditions UACl and UAC3 are independent.

\section{PROOF}

Part 1. The proof uses the following claim.

CLAIM When URC1, URC2, UM1 and UM2 hold then we have one of the following: 
1. $(\alpha, \beta) \succ^{*}(\beta, \beta) \succ^{*}(\beta, \alpha)$, for all $\alpha, \beta \in \Gamma$ such that $(\alpha, \beta) \succ^{* *}(\beta, \beta)$,

2. $(\alpha, \beta) \succ^{*}(\beta, \beta)$ and $(\beta, \beta) \sim^{*}(\beta, \alpha)$, for all $\alpha, \beta \in \Gamma$ such that $(\alpha, \beta) \succ^{* *}(\beta, \beta)$,

3. $(\alpha, \beta) \sim^{*}(\beta, \beta)$ and $(\beta, \beta) \succ^{*}(\beta, \alpha)$, for all $\alpha, \beta \in \Gamma$ such that $(\alpha, \beta) \succ^{* *}(\beta, \beta)$,

\section{PROOF OF THE CLAIM}

Using part 3 of lemma 4 and part 8 of lemma 8 , we know that $\succsim^{* *}$ is a weak order having at most three distinct equivalence classes. Let $\alpha, \beta \in \Gamma$ be such that $(\alpha, \beta) \succ^{* *}(\beta, \beta)$. By construction, we have either $(\alpha, \beta) \succ^{*}(\beta, \beta)$ or $(\beta, \beta) \succ^{*}(\beta, \alpha)$. There are three cases to examine.

1. Suppose first that $(\alpha, \beta) \succ^{*}(\beta, \beta)$ and $(\beta, \beta) \succ^{*}(\beta, \alpha)$. Consider $\gamma, \delta \in \Gamma$ such that $(\gamma, \delta) \succ^{* *}(\delta, \delta)$. If either $(\gamma, \delta) \sim^{*}(\delta, \delta)$ or $(\delta, \gamma) \sim^{*}(\delta, \delta)$, it is easy to see, using the independence of $\succsim$ and the definition of $\succsim^{* *}$, that we must have:

$$
(\alpha, \beta) \succ^{* *}(\gamma, \delta) \succ^{* *}(\beta, \beta) \succ^{* *}(\delta, \gamma) \succ^{* *}(\beta, \alpha),
$$

violating the fact that $\sim^{* *}$ has at most three distinct equivalence classes. Hence we have, for all $\gamma, \delta \in \Gamma$ such that $(\gamma, \delta) \succ^{* *}(\delta, \delta),(\gamma, \delta) \succ^{*}(\delta, \delta)$ and $(\delta, \delta) \succ^{*}(\delta, \gamma)$.

2. Suppose that $(\alpha, \beta) \succ^{*}(\beta, \beta)$ and $(\beta, \beta) \sim^{*}(\beta, \alpha)$ and consider any $\gamma, \delta \in \Gamma$ such that $(\gamma, \delta) \succ^{* *}(\delta, \delta)$. If $(\gamma, \delta) \succ^{*}(\delta, \delta)$ and $(\delta, \delta) \succ^{*}(\delta, \gamma)$, we have, using the independence of $\succsim$ and the definition of $\succsim^{* *}$,

$$
(\gamma, \delta) \succ^{* *}(\alpha, \beta) \succ^{* *}(\beta, \beta) \succ^{* *}(\beta, \alpha) \succ^{* *}(\delta, \gamma),
$$

violating the fact that $\sim^{* *}$ has at most three distinct equivalence classes. If $(\gamma, \delta) \sim^{*}$ $(\delta, \delta)$ and $(\delta, \delta) \succ^{*}(\delta, \gamma)$, then URC2 is violated since we have $(\alpha, \beta) \succ^{*}(\gamma, \delta)$ and $(\beta, \alpha) \succ^{*}(\delta, \gamma)$. Hence, it must be true that $(\gamma, \delta) \succ^{* *}(\delta, \delta)$ implies $(\gamma, \delta) \succ^{*}$ $(\delta, \delta)$ and $(\delta, \delta) \sim^{*}(\delta, \gamma)$

3. Suppose that $(\alpha, \beta) \sim^{*}(\beta, \beta)$ and $(\beta, \beta) \succ^{*}(\beta, \alpha)$ and consider any $\gamma, \delta \in \Gamma$ such that $(\gamma, \delta) \succ^{* *}(\delta, \delta)$. If $(\gamma, \delta) \succ^{*}(\delta, \delta)$ and $(\delta, \delta) \succ^{*}(\delta, \gamma)$, we have, using the independence of $\succsim$ and the definition of $\succsim^{* *}$,

$$
(\gamma, \delta) \succ^{* *}(\alpha, \beta) \succ^{* *}(\beta, \beta) \succ^{* *}(\beta, \alpha) \succ^{* *}(\delta, \gamma),
$$

violating the fact that $\sim^{* *}$ has at most three distinct equivalence classes. If $(\gamma, \delta) \succ^{*}$ $(\delta, \delta)$ and $(\delta, \delta) \sim^{*}(\delta, \gamma)$, then URC2 is violated since we have $(\gamma, \delta) \succ^{*}(\alpha, \beta)$ and $(\delta, \gamma) \succ^{*}(\beta, \alpha)$. Hence, it must be true that $(\gamma, \delta) \succ^{* *}(\delta, \delta)$ implies $(\gamma, \delta) \sim^{*}$ $(\delta, \delta)$ and $(\delta, \delta) \succ^{*}(\delta, \gamma)$

This proves the claim. 
We prove that $\mathrm{UAC} 1 \Rightarrow \mathrm{UAC} 2$, the proof of the reverse implication being similar. Suppose UAC2 is violated so that, for some $a, b, c, d \in \mathcal{A}$ and some $\alpha, \beta \in \Gamma$, we have $a \succsim \alpha_{i} b c \succsim \beta_{j} d, \operatorname{Not}\left[a \succsim \beta_{i} b\right], \operatorname{Not}\left[c \succsim \alpha_{j} d\right]$.

This implies $(\alpha, \beta) \succ^{*}(\alpha, \delta)$ and $(\gamma, \delta) \succ^{*}(\gamma, \beta)$, so that $(\alpha, \beta) \succ^{* *}(\alpha, \delta)$ and $(\gamma, \delta) \succ^{* *}(\gamma, \beta)$.

Because, URC1, URC2, UM1 and UM2 hold, we know that we must be in one of the cases of the above claim.

If either of the last two cases hold, ${ }^{*}$ has at most two distinct equivalence classes, so that $(\alpha, \beta) \sim^{*}(\gamma, \delta)$ and $(\alpha, \delta) \sim^{*}(\gamma, \beta)$. This implies $(\gamma, \delta) \succ^{*}(\alpha, \delta)$ and $(\alpha, \beta) \succ^{*}$ $(\gamma, \beta)$. Since UAC1 implies the right-linearity of $\succsim^{*},(\gamma, \delta) \succ^{*}(\alpha, \delta)$ implies $(\gamma, \beta) \succsim^{*}$ $(\alpha, \beta)$, a contradiction.

Suppose that the first case holds true. We distinguish several subcases.

1. If both $(\alpha, \beta)$ and $(\gamma, \delta)$ belong to the middle equivalence class of $\succsim^{*}$, we have $\left[(\alpha, \beta) \sim^{*}(\gamma, \delta)\right] \succ^{*}\left[(\alpha, \delta) \sim^{*}(\gamma, \beta)\right]$. As shown above, this leads to a contradiction.

2. Suppose that both $(\alpha, \beta)$ and $(\gamma, \delta)$ belong to the first equivalence class of $\succsim^{*}$. We therefore have $(\alpha, \beta) \sim^{*}(\gamma, \delta),(\alpha, \beta) \succ^{*}(\alpha, \delta)$ and $(\gamma, \delta) \succ^{*}(\gamma, \beta)$. This implies $(\alpha, \beta) \succ^{*}(\gamma, \beta)$. Using UAC1, we have $(\alpha, \delta) \succsim^{*}(\gamma, \delta)$, a contradiction.

3. Suppose that $(\alpha, \beta)$ belongs to the first equivalence class of $\succsim^{*}$ and $(\gamma, \delta)$ belongs to the central class of $\succsim^{*}$. This implies, using the reversibility of $\succsim^{* *}$ and the fact that it has at most three equivalence classes, $\left[(\alpha, \beta) \sim^{*}(\beta, \gamma)\right] \succ^{*}\left[(\gamma, \delta) \sim^{*}(\delta, \gamma)\right] \succ^{*}$ $\left[(\gamma, \beta) \sim^{*}(\beta, \alpha)\right]$. Hence, we have $(\beta, \gamma) \succ^{*}(\delta, \gamma)$ and using UAC1, we have $(\beta, \alpha) \succsim^{*}(\delta, \alpha)$, a contradiction.

Part 2: see examples 11 and 12 in appendix

This leads to a characterization of LD relations in which $\mathcal{S}$ is a semiorder.

\section{Theorem 4}

Let $\succsim$ be a binary relation on $\mathcal{A}$. Then $\succsim$ is a $L D$ relation having a representation $\langle\unrhd, \mathcal{S}\rangle$ in which $\mathcal{S}$ is a semiorder iff it is reflexive and satisfies URC2, UM1, UM2, UAC1 and UAC3.

\section{PROOF}

The proof of theorem 4 follows from combining lemmas 9, 10 and 11 with the results in section 5 . 
Let us finally mention that in our definition of LD relations in section 3, the only remarkable property imposed on $\unrhd$ is monotonicity w.r.t. inclusion. In most instances, we would expect $\unrhd$ to be transitive as well. It is easy to devise conditions that imply the transitivity of $\unrhd$. We leave the details to the interested reader.

\section{Appendices}

\section{A Examples related to model (UM)}

\section{Example 2 (URC2, Not [URC1])}

Let $\Gamma=\{\alpha, \beta, \gamma\}$ and $N=\{1,2\}$. Let $\succsim$ on $\mathcal{A}$ identical to $\mathcal{A}^{2}$ except that, using obvious notation, $\operatorname{Not}\left[\alpha_{1} \gamma_{2} \succsim \beta_{1} \alpha_{2}\right]$ and $\operatorname{Not}\left[\gamma_{1} \alpha_{2} \succsim \alpha_{1} \beta_{2}\right]$.

It is easy to see that $\succsim$ is complete (and, hence, reflexive). It violates URC1 since $\alpha_{1} \alpha_{2} \succsim \beta_{1} \beta_{2}$ and $\gamma_{1} \gamma_{2} \succsim \alpha_{1} \alpha_{2}$ but neither $\alpha_{1} \gamma_{2} \succsim \beta_{1} \alpha_{2}$ nor $\gamma_{1} \alpha_{2} \succsim \alpha_{1} \beta_{2}$.

It is not difficult to check that we have:

- $[(\alpha, \alpha),(\beta, \beta),(\gamma, \gamma),(\alpha, \gamma),(\beta, \alpha),(\beta, \gamma),(\gamma, \beta)] \succ^{*}(\alpha, \beta)$ and

- $[(\alpha, \alpha),(\beta, \beta),(\gamma, \gamma),(\alpha, \gamma),(\beta, \alpha),(\beta, \gamma),(\gamma, \beta)] \succ^{*}(\gamma, \alpha)$,

while $(\alpha, \beta)$ and $(\gamma, \alpha)$ are incomparable in terms of $\succsim^{*}$. Using part 2 of lemma 4 , it is easy to check that $\succsim$ satisfies URC2.

Example 3 (URC1, Not [URC2])

Let $\Gamma=\{\alpha, \beta\}$ and $N=\{1,2\}$. Let $\succsim$ on $\mathcal{A}$ be such that:

$$
a \succsim b \Leftrightarrow p\left(a_{1}, b_{1}\right)+p\left(a_{2}, b_{2}\right) \geq 0,
$$

where $p$ is a real valued function on $\Gamma^{2}$ defined by the following table (to be read from line to column):

\begin{tabular}{r|rr}
$p$ & $\alpha$ & $\beta$ \\
\hline$\alpha$ & 0 & -1 \\
$\beta$ & 1 & 1
\end{tabular}

It is easy to see that $\succsim$ is complete (and hence, reflexive) and satisfies URC1 (we have: $\left.\left[(\beta, \beta) \sim^{*}(\beta, \alpha)\right] \succ^{*}(\alpha, \alpha) \succ^{*}(\alpha, \beta)\right)$. The relation $\succsim$ is not independent since $\beta_{1} \alpha_{2} \succsim$ $\beta_{1} \beta_{2}$ but $\left.\operatorname{Not}\left[\alpha_{1} \alpha_{2}\right) \succsim \alpha_{1} \beta_{2}\right]$. Hence, URC2 is violated in view of part 5 of lemma 4 . 


\section{B Examples related to $L D$ relations}

\section{Example 4 (URC1, URC2, UM2, $\operatorname{Not}[\mathrm{UM1}]$ )}

Let $\Gamma=\{\alpha, \beta, \gamma\}$ and $N=\{1,2\}$. Let $\succsim$ on $\mathcal{A}$ be such that:

$$
a \succsim b \Leftrightarrow p_{1}\left(a_{1}, b_{1}\right)+p_{2}\left(a_{2}, b_{2}\right) \geq 0
$$

where $p_{1}$ and $p_{2}$ are real valued functions on $\Gamma^{2}$ defined by the following table:

\begin{tabular}{|c|c|c|c|c|c|c|c|}
\hline$p_{1}$ & $\alpha$ & $\beta$ & $\gamma$ & $p_{2}$ & $\alpha$ & $\beta$ & $\gamma$ \\
\hline$\alpha$ & 0 & 4 & 0 & $\alpha$ & 0 & 0 & 0 \\
\hline$\beta$ & 0 & 0 & 0 & $\beta$ & -3 & 0 & 0 \\
\hline$\gamma$ & 0 & 0 & 0 & $\gamma$ & -3 & -3 & 0 \\
\hline
\end{tabular}

The relation $\succsim$ is clearly complete. It is not difficult to see that $\succsim^{*}$ is such that:

$$
(\alpha, \beta) \succ^{*}[(\alpha, \alpha),(\beta, \beta),(\gamma, \gamma),(\alpha, \gamma),(\beta, \gamma)] \succ^{*}[(\beta, \alpha),(\gamma, \alpha),(\gamma, \beta)]
$$

This shows, in view of lemma 4, that URC1 and URC2 are satisfied. It is easy to check that (7) holds, so that the same is true for UM2. We have $(\alpha, \gamma) \succ^{*}(\gamma, \alpha)$ but $\operatorname{Not}\left[(\alpha, \gamma) \succsim^{*}(\alpha, \beta)\right]$. This shows that (6) is violated. Since URC2 holds, this shows that UM1 is violated in view of part 5 of lemma 6.

Example 5 (URC1, URC2, UM1, Not[UM2])

Let $\Gamma=\{\alpha, \beta, \gamma\}$ and $N=\{1,2\}$. Let $\succsim$ on $\mathcal{A}$ be such that:

$$
a \succsim b \Leftrightarrow g\left(p_{1}\left(a_{1}, b_{1}\right)+p_{2}\left(a_{2}, b_{2}\right)\right) \geq 0,
$$

\begin{tabular}{|c|c|c|c|c|c|c|}
\hline$p_{1}$ & $\alpha$ & $\beta$ & $\gamma$ & $p_{2}$ & $\alpha$ & $\beta$ \\
\hline$\alpha$ & 0 & 2 & 2 & $\alpha$ & 0 & 0 \\
\hline$\beta$ & -2 & 0 & 2 & $\beta$ & -2 & 0 \\
\hline$\gamma$ & -4 & -2 & 0 & $\gamma$ & -2 & -2 \\
\hline
\end{tabular}

where $p_{1}$ and $p_{2}$ are real valued functions on $\Gamma^{2}$ defined by the following table:

and $g$ is such that:

$$
g(x)=\left\{\begin{array}{l}
x \text { if }|x|>2 \\
0 \text { otherwise }
\end{array}\right.
$$

The relation $\succsim$ is clearly complete. It is not difficult to see that ${ }^{*}$ is such that:

$$
[(\alpha, \alpha),(\beta, \beta),(\gamma, \gamma),(\alpha, \beta),(\alpha, \gamma),(\beta, \gamma)] \succ^{*}[(\beta, \alpha),(\gamma, \beta)] \succ^{*}(\gamma, \alpha) .
$$


This shows, in view of lemma 4, that URC1 and URC2 are satisfied. It is easy to check that (6) holds, so that the same is true for UM1. We have $(\alpha, \beta) \succ^{*}(\beta, \alpha)$ but $\operatorname{Not}\left[(\gamma, \alpha) \succsim^{*}(\beta, \alpha)\right]$. This shows that (7) is violated. Since URC1 holds, this shows that $\mathrm{UM} 2$ is violated in view of part 6 of lemma 6 .

\section{Example 6 (URC1, UM1, UM2, Not[URC2])}

Let $\Gamma=\{\alpha, \beta\}$ and $N=\{1,2\}$. Let $\succsim$ on $\mathcal{A}$ be identical $\mathcal{A}^{2}$ except that $\operatorname{Not}\left[\beta_{1} \beta_{2} \succsim \alpha_{1} \alpha_{2}\right]$ and $\operatorname{Not}\left[\beta_{1} \beta_{2} \succsim \alpha_{1} \beta_{2}\right]$. This relation is clearly complete. It is not independent, so that URC2 is violated in view of lemma 4. We have: $[(\alpha, \alpha),(\alpha, \beta)] \succ^{*}(\beta, \beta) \succ^{*}(\beta, \alpha)$. Since $\succsim^{*}$ is complete, URC1 holds. In view of parts 1 and 2 of lemma 6 , we know that (6) and (7) hold. Hence, UM1 and UM2 hold.

\section{Example 7 (URC2, UM1, UM2, Not [URC1])}

Let $\Gamma=\{\alpha, \beta, \gamma\}$ and $N=\{1,2,3\}$. Let $\succsim$ on $\mathcal{A}$ be identical to $\mathcal{A}^{2}$ except that the following 25 relations are missing: $\alpha_{1} \alpha_{2} \alpha_{3} \succsim \gamma_{1} \alpha_{2} \gamma_{3}, \alpha_{1} \alpha_{2} \alpha_{3} \succsim \gamma_{1} \beta_{2} \gamma_{3}, \alpha_{1} \alpha_{2} \alpha_{3} \succsim$ $\gamma_{1} \gamma_{2} \gamma_{3}, \alpha_{1} \beta_{2} \alpha_{3} \succsim \alpha_{1} \alpha_{2} \gamma_{3}, \alpha_{1} \beta_{2} \alpha_{3} \succsim \beta_{1} \alpha_{2} \gamma_{3}, \alpha_{1} \beta_{2} \alpha_{3} \succsim \gamma_{1} \alpha_{2} \gamma_{3}, \alpha_{1} \beta_{2} \alpha_{3} \succsim \gamma_{1} \beta_{2} \gamma_{3}$, $\alpha_{1} \beta_{2} \alpha_{3} \succsim \gamma_{1} \gamma_{2} \gamma_{3}, \alpha_{1} \gamma_{2} \alpha_{3} \succsim \gamma_{1} \alpha_{2} \gamma_{3}, \alpha_{1} \gamma_{2} \alpha_{3} \succsim \gamma_{1} \beta_{2} \gamma_{3}, \alpha_{1} \gamma_{2} \alpha_{3} \succsim \gamma_{1} \gamma_{2} \gamma_{3}, \beta_{1} \beta_{2} \alpha_{3} \succsim$ $\alpha_{1} \alpha_{2} \alpha_{3}, \beta_{1} \beta_{2} \alpha_{3} \succsim \alpha_{1} \alpha_{2} \beta_{3}, \beta_{1} \beta_{2} \alpha_{3} \succsim \alpha_{1} \alpha_{2} \gamma_{3}, \beta_{1} \beta_{2} \alpha_{3} \succsim \beta_{1} \alpha_{2} \gamma_{3}, \beta_{1} \beta_{2} \alpha_{3} \succsim \gamma_{1} \alpha_{2} \gamma_{3}$, $\beta_{1} \beta_{2} \beta_{3} \succsim \alpha_{1} \alpha_{2} \alpha_{3}, \beta_{1} \beta_{2} \beta_{3} \succsim \alpha_{1} \alpha_{2} \beta_{3}, \beta_{1} \beta_{2} \beta_{3} \succsim \alpha_{1} \alpha_{2} \gamma_{3}, \beta_{1} \beta_{2} \gamma_{3} \succsim \alpha_{1} \alpha_{2} \alpha_{3}, \beta_{1} \beta_{2} \gamma_{3} \succsim$ $\alpha_{1} \alpha_{2} \beta_{3}, \beta_{1} \beta_{2} \gamma_{3} \succsim \alpha_{1} \alpha_{2} \gamma_{3}, \gamma_{1} \beta_{2} \alpha_{3} \succsim \alpha_{1} \alpha_{2} \gamma_{3}, \gamma_{1} \beta_{2} \alpha_{3} \succsim \beta_{1} \alpha_{2} \gamma_{3}$ and $\gamma_{1} \beta_{2} \alpha_{3} \succsim \gamma_{1} \alpha_{2} \gamma_{3}$.

It is not difficult to check that $\succsim$ is complete. We have:

$$
\begin{aligned}
& {[(\alpha, \alpha),(\beta, \beta),(\gamma, \gamma),(\beta, \gamma),(\gamma, \alpha),(\gamma, \beta),(\gamma, \beta)] \succ^{*}(\alpha, \gamma) \text { and }} \\
& {[(\alpha, \alpha),(\beta, \beta),(\gamma, \gamma),(\beta, \gamma),(\gamma, \alpha),(\gamma, \beta),(\gamma, \beta)] \succ^{*}(\beta, \alpha),}
\end{aligned}
$$

while $(\alpha, \gamma)$ and $(\beta, \alpha)$ are nor comparable in terms of $\succsim^{*}$. This shows that URC1 is violated. Using part 2 of lemma 4, it is easy to check that URC2 holds. Using part 1 of lemma 6 , it is easy to check that (6) holds. In view of part 3 of lemma 6 , this shows that UM1 is satisfied. It remains to check that UM2 holds.

It is not difficult to check that $\beta_{2} a \succsim \alpha_{2} b$ implies $\tau_{2} a \succsim \sigma_{2} b$, for all $a, b \in \mathcal{A}$ and all $(\tau, \sigma) \in \Gamma^{2}$. Furthermore, for all $(\tau, \sigma),(\chi, \psi) \in \Gamma^{2} \backslash(\beta, \alpha), \chi_{2} a \succsim \psi_{2} b \Leftrightarrow \tau_{2} a \succsim \sigma_{2} b$. Similarly, it is easy to check that $\alpha_{3} a \succsim \gamma_{3} b$ implies $\tau_{3} a \succsim \sigma_{3} b$, for all $a, b \in \mathcal{A}$ and all $(\tau, \sigma) \in \Gamma^{2}$. Furthermore, for all $(\tau, \sigma),(\chi, \psi) \in \Gamma^{2} \backslash(\alpha, \gamma), \chi_{3} a \succsim \psi_{3} b \Leftrightarrow \tau_{3} a \succsim \sigma_{3} b$.

The two premises of UM2 are that $\tau_{i} a \succsim \sigma_{i} b$ and $\sigma_{j} c \succsim \tau_{j} d$. The three possible conclusions of UM2 are that $\sigma_{i} a \succsim \tau_{i} b$ or $\chi_{i} a \succsim \psi_{i} b$ or $\chi_{j} c \succsim \psi_{j} d$.

Suppose first that $(\tau, \sigma)$ is distinct from $(\gamma, \alpha)$ and $(\alpha, \beta)$. In this case, we know that $(\sigma, \tau) \succsim^{*}(\tau, \sigma)$, so that $\tau_{i} a \succsim \sigma_{i} b$ implies $\sigma_{i} a \succsim \tau_{i} b$. Hence, the first conclusion of UM2 will hold.

Suppose henceforth that $(\tau, \sigma)=(\gamma, \alpha)$. If $i=2$, we know that $\gamma_{2} a \succsim \alpha_{2} b \Leftrightarrow$ $\alpha_{2} a \succsim \gamma_{2} b$, so that the first conclusion of UM2 will hold. 
Suppose that $i=3$. If $j=3$, the second premise of UM2 becomes $\alpha_{3} c \succsim \gamma_{3} d$. This implies $\gamma_{3} c \succsim \alpha_{3} d$ so that the last conclusion of UM2 will hold. A similar reasoning shows that the last conclusion of UM2 will hold if $j=1$. Suppose that $j=2$. The two premises of UM2 are that $\gamma_{3} a \succsim \alpha_{3} b$ and $\alpha_{2} c \succsim \gamma_{2} d$. The three desired conclusions are that either $\gamma_{3} a \succsim \alpha_{3} b$ or $\chi_{3} a \succsim \psi_{3} b$ or $\chi_{2} c \succsim \psi_{2} d$. If $(\chi, \psi)$ is distinct from $(\beta, \alpha)$, we know that $\alpha_{2} c \succsim \gamma_{2} d \Leftrightarrow \chi_{2} c \succsim \psi_{2} d$ so that the last conclusion of UM2 will hold. Now if $(\chi, \psi)=(\beta, \alpha)$, we have that $\beta_{3} a \succsim \alpha_{3} b$ so that the second conclusion of UM2 holds.

Suppose that $i=1$. If $(\chi, \psi)$ is distinct from $(\beta, \alpha), \gamma_{1} a \succsim \alpha_{1} b$ will imply $\chi_{1} a \succsim \psi_{1} b$, so that the second conclusion of UM2 will hold. If $(\chi, \psi)=(\beta, \alpha)$, it is easy to check that there is no $a, b \in \mathcal{A}$ such that $\gamma_{1} a \succsim \alpha_{1} b, \operatorname{Not}\left[\alpha_{1} a \succsim \gamma_{1} b\right]$ and $\operatorname{Not}\left[\beta_{1} a \succsim \alpha_{1} b\right]$. This shows that UM2 cannot be violated.

Hence, we have shown that $\mathrm{UM} 2$ holds if $(\tau, \sigma)=(\gamma, \alpha)$. A similar reasoning shows that UM2 holds if $(\tau, \sigma)=(\alpha, \beta)$.

\section{Examples related to model $\left(\mathrm{UM}^{*}\right)$}

Throughout the remaining examples, we use the following notation:

$$
\begin{gathered}
\alpha \succsim^{ \pm} \beta \Leftrightarrow\left[(\alpha, \gamma) \succsim^{*}(\beta, \gamma) \text { and }(\delta, \beta) \succsim^{*}(\delta, \alpha), \forall \gamma, \delta \in \Gamma\right], \\
\alpha \succsim^{+} \beta \Leftrightarrow\left[(\alpha, \gamma) \succsim^{*}(\beta, \gamma), \forall \gamma \in \Gamma\right], \\
\alpha \succsim^{-} \beta \Leftrightarrow\left[(\delta, \beta) \succsim^{*}(\delta, \alpha), \forall \delta \in \Gamma\right] .
\end{gathered}
$$

The reader will easily check that:

$$
\begin{aligned}
& \text { UAC } 1 \Leftrightarrow \succsim^{+} \text {is complete, } \\
& \mathrm{UAC} 2 \Leftrightarrow \succsim^{-} \text {is complete, } \\
& \mathrm{UAC} 3 \Leftrightarrow\left[\alpha \succ^{+} \beta \Rightarrow \operatorname{Not}\left[\beta \succ^{-} \alpha\right]\right] .
\end{aligned}
$$

It is also interesting to note that:

$$
\begin{gathered}
\alpha \succsim^{+} \beta \Leftrightarrow\left[\beta_{i} c \succsim d \Rightarrow \alpha_{i} c \succsim d, \forall c, d \in \mathcal{A}\right], \\
\alpha \succsim^{-} \beta \Leftrightarrow\left[d \succsim \alpha_{i} c \Rightarrow d \succsim \beta_{i} c, \forall c, d \in \mathcal{A}\right], \\
\alpha \succsim^{ \pm} \beta \Leftrightarrow\left[\alpha \succsim^{+} \beta \text { and } \alpha \succsim^{-} \beta\right] .
\end{gathered}
$$

Example 8 (URC1, URC2, UAC2, UAC3, $\operatorname{Not}$ [UAC1])

Let $\Gamma=\{\alpha, \beta, \gamma, \delta\}$ and $N=\{1,2\}$. Let $\succsim$ on $\mathcal{A}$ be such that:

$$
a \succsim b \Leftrightarrow g\left(p\left(a_{1}, b_{1}\right)+p\left(a_{2}, b_{2}\right)\right) \geq 0
$$

where $p$ is a real valued function on $\Gamma^{2}$ defined by the following table: 


\begin{tabular}{r|rrrr}
$p$ & $\alpha$ & $\beta$ & $\gamma$ & $\delta$ \\
\hline$\alpha$ & 0 & -3 & -1 & 2 \\
$\beta$ & 3 & 0 & 1 & 2 \\
$\gamma$ & 1 & -1 & 0 & 2 \\
$\delta$ & -2 & -2 & -2 & 0
\end{tabular}

and $g$ is such that:

$$
g(x)=\left\{\begin{array}{l}
x \text { if }|x|>2 \\
0 \text { otherwise }
\end{array}\right.
$$

The relation $\succsim$ is clearly complete and satisfies URC1 and URC2. It is not difficult to check that we have:

$$
\beta \succ^{-} \gamma \succ^{-} \alpha \succ^{-} \delta
$$

We have $\beta \succ^{+} \gamma, \gamma \succ^{+} \alpha$ and $\gamma \succ^{+} \delta$ but neither $\alpha \succsim^{+} \delta$ (because $\delta_{1} \alpha_{2} \succsim \beta_{1} \alpha_{2}$ but $\left.\operatorname{Not}\left[\alpha_{1} \alpha_{2} \succsim \beta_{1} \alpha_{2}\right]\right)$ nor $\delta \succsim^{+} \alpha$ (because $\alpha_{1} \alpha_{2} \succsim \alpha_{1} \gamma_{2}$ but $\operatorname{Not}\left[\delta_{1} \alpha_{2} \succsim \alpha_{1} \gamma_{2}\right]$ ). This shows that UAC2 and UAC3 hold but that UAC1 is violated.

Example 9 (URC1, URC2, UAC1, UAC3, Not[UAC2])

Let $\Gamma=\{\alpha, \beta, \gamma, \delta\}$ and $N=\{1,2\}$. Let $\succsim$ on $\mathcal{A}$ be such that:

$$
a \succsim b \Leftrightarrow g\left(p\left(a_{1}, b_{1}\right)+p\left(a_{2}, b_{2}\right)\right) \geq 0
$$

where $p$ is a real valued function on $\Gamma^{2}$ defined by the following table:

\begin{tabular}{r|rrrr}
$p$ & $\alpha$ & $\beta$ & $\gamma$ & $\delta$ \\
\hline$\alpha$ & 0 & 3 & 1 & -2 \\
$\beta$ & -3 & 0 & -1 & -2 \\
$\gamma$ & -1 & 1 & 0 & -2 \\
$\delta$ & 2 & 2 & 2 & 0
\end{tabular}

and $g$ is as in example 8 .

The relation $\succsim$ is clearly complete and satisfies URC1 and URC2. Observe that $p$ is defined via the transposition of the table used in example 8. This interchanges the roles of UAC1 and UAC2. In fact it is not difficult to see that we have:

$$
\delta \succ^{+} \alpha \succ^{+} \gamma \succ^{+} \beta
$$

We have: $\delta \succ^{-} \gamma, \alpha \succ^{-} \gamma, \gamma \succ^{-} \beta$ but neither $\alpha \succsim^{-} \delta$ nor $\delta \succsim^{-} \alpha$. This shows that $\mathrm{UAC} 1$ and UAC3 hold but that UAC2 is violated. 


\section{Example 10 (URC1, URC2, UAC1, UAC2, Not[UAC3])}

Let $\Gamma=\{\alpha, \beta, \gamma, \delta\}$ and $N=\{1,2\}$. Let $\succsim$ on $\mathcal{A}$ be such that:

$$
a \succsim b \Leftrightarrow g\left(p\left(a_{1}, b_{1}\right)+p\left(a_{2}, b_{2}\right)\right) \geq 0
$$

where $p$ is a real valued function on $\Gamma^{2}$ defined by the following table:

\begin{tabular}{r|rrrr}
$p$ & $\alpha$ & $\beta$ & $\gamma$ & $\delta$ \\
\hline$\alpha$ & 0 & -5 & 0 & -2 \\
$\beta$ & 5 & 0 & 1 & 2 \\
$\gamma$ & 0 & -1 & 0 & 0 \\
$\delta$ & 2 & -2 & 0 & 0
\end{tabular}

and $g$ is as in example 8 .

The relation $\succsim$ is clearly complete and satisfies URC1 and URC2. We have:

$$
\begin{aligned}
& \beta \succ^{+} \gamma \succ^{+} \delta \succ^{+} \alpha \text { and } \\
& \beta \succ^{-} \delta \succ^{-} \gamma \succ^{-} \alpha .
\end{aligned}
$$

This shows that UAC1 and UAC2 hold but that UAC3 is violated since $\gamma \succ^{+} \delta$ but $\delta \succ^{-} \gamma$.

\section{Examples related to $L D$ relations in which $\mathcal{S}$ is a semiorder}

Example 11 (URC1, URC2, UM1, UM2, UAC1, UAC2, Not[UAC3])

Let $\Gamma=\{\alpha, \beta, \gamma, \delta\}$ and $N=\{1,2\}$. Let $\succsim$ on $\mathcal{A}$ be such that:

$$
a \succsim b \Leftrightarrow g\left(p\left(a_{1}, b_{1}\right)+p\left(a_{2}, b_{2}\right)\right) \geq 0
$$

where $p$ is a real valued function on $\Gamma^{2}$ defined by the following table:

\begin{tabular}{r|rrrr}
$p$ & $\alpha$ & $\beta$ & $\gamma$ & $\delta$ \\
\hline$\alpha$ & 0 & -2 & 0 & -2 \\
$\beta$ & 2 & 0 & 0 & 2 \\
$\gamma$ & 0 & 0 & 0 & 0 \\
$\delta$ & 2 & -2 & 0 & 0
\end{tabular}

and $g$ is as in example 8 . 
The relation $\succsim$ is clearly complete and satisfies URC1 and URC2. Since $p$ takes 3 distinct values, it is easy to see that UM1 and UM2 holds. We have:

$$
\begin{aligned}
& {[\beta, \gamma] \succ^{+} \delta \succ^{+} \alpha \text { and }} \\
& \beta \succ^{-} \delta \succ^{-}[\gamma, \alpha] .
\end{aligned}
$$

This shows that UAC1 and UAC2 hold but that UAC3 is violated since $\gamma \succ^{+} \delta$ but $\delta \succ^{-} \gamma$.

Example 12 (URC1, URC2, UM1, UM2, UAC3, Not[UAC1], $\operatorname{Not}$ [UAC2])

Let $\Gamma=\{\alpha, \beta, \gamma, \delta\}$ and $N=\{1,2\}$. Let $\succsim$ on $\mathcal{A}$ be such that:

$$
a \succsim b \Leftrightarrow g\left(p\left(a_{1}, b_{1}\right)+p\left(a_{2}, b_{2}\right)\right) \geq 0
$$

where $p$ is a real valued function on $\Gamma^{2}$ defined by the following table:

\begin{tabular}{r|rrrr}
$p$ & $\alpha$ & $\beta$ & $\gamma$ & $\delta$ \\
\hline$\alpha$ & 0 & -2 & -2 & 2 \\
$\beta$ & 2 & 0 & 0 & 0 \\
$\gamma$ & 2 & 0 & 0 & 2 \\
$\delta$ & -2 & 0 & -2 & 0
\end{tabular}

and $g$ is as in example 8 .

The relation $\succsim$ is clearly complete and satisfies URC1 and URC2. Since $p$ takes 3 distinct values, it is easy to see that UM1 and UM2 holds. It is easy to see that: $\beta \sim^{+} \gamma$, $\beta \succ^{+} \alpha, \beta \succ^{+} \delta, \gamma \succ^{+} \alpha, \gamma \succ^{+} \delta$, but neither $\alpha \succsim^{+} \delta$ nor $\delta \succsim^{+} \alpha$. Similarly we obtain: $\gamma \succ^{-} \alpha, \gamma \succ^{-} \beta, \gamma \succ^{-} \delta, \alpha \succ^{-} \delta, \beta \succ^{-} \delta$ but neither $\alpha \succsim^{-} \beta$ nor $\beta \succsim^{-} \alpha$. Hence UAC3 holds but UAC1 and UAC2 are violated.

\section{References}

Allais, M. (1953). Le comportement de l'homme rationnel devant le risque : critique des postulats et axiomes de l'école américaine. Econometrica, 21:503-46.

Boutilier, C. (1994). Toward a logic for qualitative decision theory. In: J. Doyle, E. Sandewall, and P. Torasso (Eds.) Proceedings of the 4th International Conference on Principles of Knowledge Representation and Reasoning (KR'94), pp. 75-86. Morgan Kaufmann Publishers, San Mateo, CA.

Bouyssou, D. (1986). Some remarks on the notion of compensation in MCDM. European Journal of Operational Research, 26:150-160. 
Bouyssou, D. (1992). On some properties of outranking relations based on a concordancediscordance principle. In: L. Duckstein, A. Goicoechea, and S. Zionts (Eds.) Multiple criteria decision making, pp. 93-106. Springer-Verlag, Berlin.

Bouyssou, D. and Pirlot, M. (2002). Nontransitive decomposable conjoint measurement. Journal of Mathematical Psychology, 46(6):677-703.

Bouyssou, D. and Pirlot, M. (2004a). 'Additive difference' models without additivity and subtractivity. Journal of Mathematical Psychology, 48(4):263-291.

Bouyssou, D. and Pirlot, M. (2004b). A characterization of concordance relations. Forthcoming in European Journal of Operational Research, available at www . lamsade. dauphine.fr/ bouyssou/.

Bouyssou, D. and Pirlot, M. (2004c). A note on Wakker's cardinal coordinate independence. Mathematical Social Sciences, 48(1):11-22.

Bouyssou, D. and Vansnick, J.-C. (1986). Noncompensatory and generalized noncompensatory preference structures. Theory and Decision, 21:251-266.

Brafman, R. and Tennenholtz, M. (1997). Modeling agents as qualitative decision makers. Artificial Intelligence, 94:217-268.

Brafman, R. and Tennenholtz, M. (2000). On the axiomatization of qualitative decision criteria. Journal of the ACM, 47:452-482.

Campbell, D. E. and Kelly, J. S. (2002). Impossibility theorems in the arrovian framework. In: K. J. Arrow, A. K. Sen, and K. Suzumura (Eds.) Handbook of Social Choice and Welfare, vol. 1, pp. 35-94. North-Holland, Amsterdam.

Chew, S. H. and Karni, E. (1994). Choquet expected utility with a finite state space: Commutativity and act-independence. Journal of Economic Theory, 62(2):469-479.

Chu, F. C. and Halpern, J. Y. (2003). Great expectations. Part I: On the customizability of generalized expected utility. In: Proceedings of 18th International Joint Conference on Artificial Intelligence (IJCAI-03), pp. 297-302. Acapulco, Mexico.

Doignon, J.-P., Monjardet, B., Roubens, M., and Vincke, Ph. (1988). Biorder families, valued relations and preference modelling. Journal of Mathematical Psychology, 30:435-480.

Doyle, J. and Thomason, R. H. (1999). Background to qualitative decision theory. AI Magazine, 20(2):55-68. 
Dubois, D., Fargier, H., and Perny, P. (2003a). Qualitative decision theory with preference relations and comparative uncertainty: An axiomatic approach. Artificial Intelligence, 148:219-260.

Dubois, D., Fargier, H., Perny, P., and Prade, H. (2002). Qualitative decision theory: From Savage's axioms to nonmonotonic reasoning. Journal of the ACM, 49(4):455-495.

Dubois, D., Fargier, H., Perny, P., and Prade, H. (2003b). A characterization of generalized concordance rules in multicriteria decision-making. International Journal of Intelligent Systems, 18(7):751-774.

Dubois, D., Fargier, H., and Prade, H. (1997). Decision-making under ordinal preferences and uncertainty. In: D. Geiger and P. P. Shenoy (Eds.) Proceedings of the 13th conference on Uncertainty in Artificial Intelligence, pp. 157-164. Morgan Kaufmann, Los Altos.

Dubois, D., Prade, H., and Sabbadin, R. (2001). Decision-theoretic foundations of qualitative possibility theory. European Journal of Operational Research, 128:459-78.

Ellsberg, D. (1961). Risk, ambiguity and the Savage axioms. Quarterly Journal of Economics, 75:643-669.

Fargier, H. and Perny, P. (1999). Qualitative decision models under uncertainty without the commensurability assumption. In: K. B. Laskey and H. Prade (Eds.) Proceedings of Uncertainty in Artificial Intelligence, pp. 188-195. Morgan Kaufmann Publishers.

Fargier, H. and Perny, P. (2001). Modélisation des préférences par une règle de concordance généralisée. In: A. Colorni, M. Paruccini, and B. Roy (Eds.) A-MCD-A, Aide Multicritère à la Décision/Multiple Criteria Decision Aid, pp. 99-115. European Commission, Joint Research Centre.

Fishburn, P. C. (1970). Utility theory for decision-making. Wiley, New York.

Fishburn, P. C. (1973). Binary choice probabilities: On the varieties of stochastic transitivity. Journal of Mathematical Psychology, 10:327-352.

Fishburn, P. C. (1975). Axioms for lexicographic preferences. Review of Economic Studies, 42:415-419.

Fishburn, P. C. (1976). Noncompensatory preferences. Synthese, 33:393-403.

Fishburn, P. C. (1978). A survey of multiattribute/multicriteria evaluation theories. In: S. Zionts (Ed.) Multicriteria problem solving, pp. 181-224. Springer-Verlag, Berlin.

Fishburn, P. C. (1982). Nontransitive measurable utility. Journal of Mathematical Psychology, 26:31-67. 
Fishburn, P. C. (1984). SSB utility theory and decision-making under uncertainty. Mathematical Social Sciences, 8(3):253-285.

Fishburn, P. C. (1988). Nonlinear preference and utility theory. Johns Hopkins University Press, Baltimore.

Fishburn, P. C. (1989). Non-transitive measurable utility for decision under uncertainty. Journal of Mathematical Economics, 18:187-207.

Fishburn, P. C. (1990). Skew symmetric additive utility with finite states. Mathematical Social Sciences, 19:103-115.

Fishburn, P. C. (1991). Nontransitive preferences in decision theory. Journal of Risk and Uncertainty, 4:113-134.

Fishburn, Peter C. and Lavalle, Irving H. (1987a). A nonlinear, nontransitive and additiveprobability model for decisions under uncertainty. The Annals of Statistics, 15(2):830 844.

Fishburn, Peter C. and Lavalle, Irving H. (1987b). State-dependent SSB utility. Economics Letters, 25(1):21-25.

Fishburn, Peter C. and Lavalle, Irving H. (1988). Context-dependent choice with nonlinear and nontransitive preferences. Econometrica, 56(5):1221-1239.

Gilboa, I. (1987). Expected utility with purely subjective non-additive probabilities. Journal of Mathematical Economics, 16:65-68.

Kahneman, D. and Tversky, A. (1979). Prospect theory: An analysis of decision under risk. Econometrica, 47:263-291.

Karni, Edi and Schmeidler, David (1991). Utility theory with uncertainty. In: W Hildebrand and H. Sonnenschein (Eds.) Handbook of mathematical economics, vol. 4, pp. 1763-1831. North-Holland, Amsterdam.

Krantz, D. H., Luce, R. D., Suppes, P., and Tversky, A. (1971). Foundations of measurement, vol. 1, Additive and polynomial representations. Academic Press, New York.

Lavalle, Irving H. and Fishburn, Peter C. (1987). Decision analysis under states-additive SSB preferences. Operations Research, 35(5):722-735.

Lehmann, D. J. (1996). Generalized qualitative probability: Savage revisited. In: Proceedings of the 12th Conference on Uncertainty in Artificial Intelligence, UAI'96, pp. 381-388. Morgan Kaufmann, San Mateo, Calif. 
Loomes, G. and Sugden, R. (1982). Regret theory: An alternative theory of rational choice under uncertainty. Economic Journal, 92:805-824.

Luce, R. D. (2000). Utility of gains and losses: Measurement-theoretical and experimental approaches. Lawrence Erlbaum Publishers, Mahwah, New Jersey.

Luce, R. D. and Raiffa, H. (1957). Games and Decisions. Wiley, New York.

Milnor, J. (1954). Games against nature. In: R. M. Thrall, C. H. Coombs, and R. L. Davis (Eds.) Decision Processes, pp. 49-59. Wiley, New York.

Nakamura, Y. (1990). Subjective expected utility with non-additive probabilities on finite state spaces. Journal of Economic Theory, 51:346-366.

Nakamura, Y. (1998). Skew-symmetric additive representations of preferences. Journal of Mathematical Economics, 30:367-387.

Saari, D. G. (1998). Connecting and resolving Sen's and Arrow's theorems. Social Choice and Welfare, 15:239-261.

Savage, L. J. (1954). The Foundations of Statistics. Wiley, New York.

Schmeidler, D. (1989). Subjective probability and expected utility without additivity. Econometrica, 57:571-587.

Sen, A. K. (1986). Social choice theory. In: K. J. Arrow and M. D. Intriligator (Eds.) Handbook of mathematical economics, vol. 3, pp. 1073-1181. North-Holland, Amsterdam.

Sugden, R. (1993). An axiomatic foundation for regret theory. Journal of Economic Theory, 60:159-180.

Tan, S.-W. and Pearl, J. (1994). Qualitative decision theory. In: AAAI 1994, Proceedings of the 12th National Conference on Artificial Intelligence, Volume 2, pp. 928-933. AAAI Press.

Vansnick, J.-C. (1986). On the problems of weights in MCDM (the noncompensatory approach). European Journal of Operational Research, 24:288-294.

Wakker, P. P. (1984). Cardinal coordinate independence for expected utility. Journal of Mathematical Psychology, 28:110-117.

Wakker, P. P. (1988). Derived strength of preference relations on coordinates. Economic Letters, 28:301-306. 
Wakker, P. P. (1989). Additive representations of preferences: A new foundation of decision analysis. Kluwer, Dordrecht.

Wakker, P. P. (1994). Separating marginal utility and probabilistic risk aversion. Theory and Decision, 36(1):1-44.

Wakker, P. P. (1996). The sure-thing principle and the comonotonic sure-thing principle: an axiomatic analysis. Journal of Mathematical Economics, 25(2):213-227.

Wakker, P. P. and Tversky, Amos (1993). An axiomatization of cumulative propect theory. Journal of Risk and Uncertainty, 7:147-176. 